\title{
Posicionamiento científico de la Asociación Mexicana de Endoscopia Gastrointestinal en procedimientos anestésicos en endoscopia
}

\author{
Diego Angulo-Molina ${ }^{1}$, Mario Peláez-Luna ${ }^{1,2 *}$, Claudia I. Blanco-Vela ${ }^{3}$, Héctor Espino-Cortés ${ }^{4}$ \\ Marco A. Chávez-Ramírez ${ }^{5}$, Rodrigo Soto-Solís ${ }^{6}$, Jessy M. Méndez-Sánchez ${ }^{7}$ \\ Luis Godínez-Martínez ${ }^{4}$, Claudia Martínez-Camacho ${ }^{8}$, Alejandra Noble-Lugo ${ }^{9}$, Jordán Zamora-Godínez ${ }^{10}$, \\ Israel I. Hernández-Ortiz'11, Daniel Torres-Rubi ${ }^{12}$, Jony Cerna-Cardona ${ }^{4}$, Omar E. Trujillo-Benavides ${ }^{13}$, \\ Irving Partida-Justo ${ }^{14}$, Andrés S. Hernández-Ángeles ${ }^{15}$ y Luis G. Valle-Gutiérrez ${ }^{11}$
}

${ }^{1}$ Unidad de Endoscopia avanzada, Centro Médico ABC, Ciudad de México; ${ }^{2}$ Departamento de Gastroenterología, Instituto Nacional de Ciencias Médicas y Nutrición Salvador Zubirán, Ciudad de México; ${ }^{3}$ Unidad de Endoscopia, Hospital Central Militar, Ciudad de México; ${ }^{4}$ Unidad de Endoscopia, Hospital Juárez de México, Ciudad de México; ${ }^{5}$ Servicio de Anestesiología, Centro Médico ABC, Ciudad de México; ${ }^{6}$ Unidad de Endoscopia, Centro Médico Nacional 20 de Noviembre, Ciudad de México; ${ }^{7}$ Servicio de Anestesiología, Hospital General de Zona N. ${ }^{\circ} 42$, Puerto Vallarta, Jal.; ${ }^{8}$ Unidad de Endoscopia, Hospital de especialidades, Centro Médico Nacional Siglo XXI, Ciudad de México; ${ }^{9}$ Departamento de

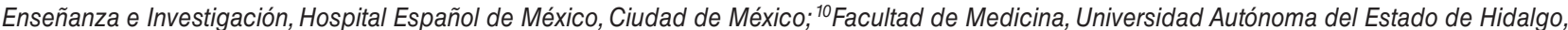
Hgo.; ${ }^{11}$ Servicio de Anestesiología, Hospital Juárez de México, Ciudad de México; ${ }^{12}$ Endoscopia, Centro Médico La Raza, Ciudad de México; ${ }^{13}$ Unidad de Endoscopia, Hospital General de Zona N. ${ }^{\circ} 42$, Puerto Vallarta, Jal.; ${ }^{14}$ Servicio de Cirugía y Endoscopia Pediátrica, Hospital Regional Lic. Adolfo López Mateos; ${ }^{15}$ Hospital Intermédica, Pachuca de Soto, Hgo. México

\section{Resumen}

La sedación en procedimientos endoscópicos tiene el objetivo de disminuir la ansiedad y las molestias que el paciente pueda experimentar producto del procedimiento, así como facilitar la exploración endoscópica. En México todo procedimiento de sedación deberá ser realizado y supervisado por un anestesiólogo certificado. Sin embargo la Asociación Mexicana de Endoscopia Gastrointestinal considera importante que los endoscopistas tengan conocimientos generales sobre las indicaciones, contraindicaciones y complicaciones de los procedimientos anestésicos en endoscopia, por lo que se realizó un posicionamiento al respecto.

Palabras clave: Sedación. Anestesia. Endoscopia gastrointestinal. Intubación orotraqueal. Posicionamiento.

\section{Anesthetic procedures in gastrointestinal endoscopy. Mexican Associaton of Gastrointestinal Endoscopy position statement}

\begin{abstract}
All endoscopic procedures should be performed under sedation. This will reduce patient's anxiety and will decrease post procedure discomfort. In Mexico, sedation must be administered and monitored by a certified anesthesiologist. Since the Asociación Mexicana de Endoscopia Gastrointestinal considers that every endoscopist should have basic knowledge of indications, contraindications and complications related to the anesthetic procedures performed during an endoscopic procedure, we developed a position statement regarding anesthetic procedures in gastrointestinal endoscopy.
\end{abstract}

Key words: Sedation. Anesthesia. Gastrointestinal endoscopy. Orotracheal intubation. Position statement.

Correspondencia:

*Mario Peláez-Luna

E-mail: mariopl@ prodigy.net.mx
Disponible en internet: 12-03-2021 Endoscopia. 2021;33(1):2-21 www.endoscopia-ameg.com
Fecha de recepción: 16-07-2020

Fecha de aceptación: 23-07-2020

DOI: 10.24875/END.M20000327 artículo open access bajo la licencia CC BY-NC-ND (http://creativecommons.org/licenses/by-nc-nd/4.0/). 


\section{Introducción}

La sedación en procedimientos endoscópicos tiene el objetivo de disminuir la ansiedad y las molestias que el paciente pueda experimentar producto del procedimiento, así como facilitar la exploración endoscópica.

Existen diferentes niveles de sedación, que van desde la analgesia hasta anestesia general, cada uno con indicaciones precisas. El tipo de sedación y los fármacos que utilizar son decisión exclusiva del anestesiólogo, de acuerdo con el procedimiento médico que se realizará y características del paciente.

Dependiendo de las legislaciones estatales y federales de cada país, la sedación junto con los cuidados preprocedimiento, transprocedimiento y posprocedimiento puede ser administrada por personal de enfermería con entrenamiento especial en endoscopia y/o en sedación, por el endoscopista o por un anestesiólogo certificado. En México, la legislación en salud indica que todo procedimiento de sedación deberá ser realizado y supervisado por un anestesiólogo certificado.

La Asociación Mexicana de Endoscopia Gastrointestinal (AMEG) y el Colegio de Profesionistas AC congregaron entre el 21 y 22 de febrero 2020 en la ciudad de San Miguel Regla (Hidalgo, México) a especialistas en endoscopia y anestesiología de instituciones públicas y privadas del país, para revisar la evidencia actual y elaborar un posicionamiento sobre los procedimientos de sedación en la práctica de la endoscopia gastrointestinal.

\section{Metodología}

Se utilizó el método Delphi. Se crearon cuatro mesas de trabajo y a cada una se le asignó un tema específico que representa cada una de las secciones de la presente guía. Seis de los participantes realizaron una búsqueda en la base de datos PubMed de artículos publicados a manera de resumen o artículo completo en los idiomas inglés y español. Se dio preferencia a revisiones sistemáticas, metaanálisis, guías de práctica clínica, consensos, ensayos clínicos controlados y cohortes, aunque no se limitó a este tipo de manuscritos.

Una vez concluida la búsqueda se elaboraron enunciados relacionados con el tema de cada mesa, mismos que fueron evaluados y votados por el comité organizador y fueron enviados a cada coordinador de mesa junto con la bibliografía seleccionada. Cada coordinador asignó aleatoriamente uno o más enunciados a cada participante, que calificó la evidencia que los apoya.
Para calificar la evidencia y seleccionar los artículos más relevantes sin importar si los resultados eran positivos o negativos se utilizó el sistema GRADE modificado (Grading of Recommendations, Assessment, Development and Evaluation).

Durante la reunión presencial los enunciados fueron presentados por los coordinadores a los miembros de sus mesas, donde se revisó el contenido y redacción de estos efectuando modificaciones en conjunto, además de discutir la pertinencia o necesidad de eliminar o agregar otros enunciados.

Se votó «en acuerdo» 0 «en desacuerdo» para cada enunciado. Cuando el acuerdo era mayor al $75 \%$ se concluyó que el enunciado permanecería sin modificaciones, y cuando el acuerdo era menor al $75 \%$ se realizaron modificaciones en contenido y redacción, sometiéndose nuevamente a votación, y así sucesivamente hasta llegar a un acuerdo mayor al $75 \%$. Aquellos enunciados con desacuerdo mayor al $75 \%$, repetidos o redundantes, fueron eliminados del proceso.

\section{Norma oficial mexicana (NOM), definiciones y medicamentos utilizados durante procedimientos anestésicos en endoscopia gastrointestinal}

\section{Norma oficial mexicana}

La AMEG recomienda que la práctica de la anestesiología durante procedimientos endoscópicos en México debe ser realizada por un anestesiólogo con certificación vigente y cédula de especialista.

La anestesiología es una rama de la medicina que mediante la aplicación de técnicas específicas y la administración de sustancias farmacológicas produce: anestesia, analgesia, inmovilidad, amnesia e inconsciencia del paciente durante el tiempo que se requiera para que se lleven a cabo procedimientos médico-quirúrgicos con fines diagnósticos, terapéuticos, paliativos, de rehabilitación o de investigación. La participación de los anestesiólogos durante la endoscopia responde a políticas institucionales y a disponibilidad de recursos humanos en cada país.

La sedación en endoscopia gastrointestinal evita molestias del paciente y facilita la exploración endoscópica. Las prácticas para suministrar sedación durante la endoscopia mediante el uso de narcóticos, benzodiacepinas y recientemente propofol han sufrido modificaciones. La administración de este último debe limitarse a anestesiólogos debido a su potencial capacidad de sedación profunda y la falta de un antagonista 
específico que pueda revertir rápidamente los efectos adversos ${ }^{1}$.

De acuerdo con la Norma Oficial Mexicana NOM-006SSA3-2011, las disposiciones para la práctica de la anestesiología deben ser cumplidas por el médico especialista en anestesiología: este debe contar con título y cédula profesional, certificado y cédula de especialización en anestesiología. Los establecimientos para la atención médica de los sectores público, social y privado donde se lleva a cabo la práctica de la anestesiología deberán contar con licencia sanitaria vigente y con el equipo mínimo necesario para garantizar su práctica en condiciones de seguridad para el paciente ${ }^{2,5}$.

\section{Definición de analgesia, sedación y anestesia. Ventajas de su uso en endoscopia}

Las guías de sedación y anestesia definen sedación como una disminución en el nivel de consciencia inducida por fármacos. La analgesia se define como la reducción o la supresión del dolor ${ }^{3-5}$.

La Norma Oficial Mexicana NOM-006-SSA3-2011 define a la anestesia general como el conjunto de técnicas utilizadas para provocar hipnosis, amnesia, analgesia, protección neurovegetativa e inmovilidad, con el objetivo de que el paciente tolere los procedimientos médicos o quirúrgicos con fines diagnósticos, terapéuticos, rehabilitatorios, paliativos o de investigación. Sedación es el estado de consciencia provocado por la administración de fármacos que permite a los pacientes tolerar procedimientos que pudieran generar molestia, conservando la capacidad de responder a las órdenes verbales y a los estímulos táctiles².

Los procedimientos endoscópicos pueden generar diversos grados de incomodidad que podrían en algunos casos impedir concluir el procedimiento. Los pacientes sometidos a esófago-gastro-duodenoscopia (EGD) sin sedación suelen presentar: náuseas, arqueo, disnea y dolor. A los que se le realiza colonoscopia principalmente se quejan de dolor y distensión abdominal ${ }^{6-8}$.

Un estudio que comparó colonoscopias realizadas con y sin sedación/analgesia observó que el uso de sedación aumentó la probabilidad de alcanzar el ciego en un $46 \%{ }^{9}$. La sedación y analgesia en endoscopia tienen como objetivo aliviar la ansiedad, evitar el malestar, dolor e incomodidad, así como producir amnesia al procedimiento, con mínimos efectos colaterales y adversos relacionados con los fármacos. Además, permite al endoscopista hacer un procedimiento seguro, cómodo y exitoso técnicamente, lo que contribuye a incrementar la calidad de la endoscopia ${ }^{3-5}$. Además de estos beneficios, la sedación no está libre de complicaciones: produce hasta el $50 \%$ de las complicaciones asociadas a la endoscopia, aunque la frecuencia de estas es baja ${ }^{10,11}$, con una tasa estimada del $0.28 \%$ durante colonoscopia y del $0.39 \%$ durante endoscopia alta $^{12}$.

\section{Niveles de sedación}

La AMEG recomienda que el nivel de sedación utilizado se elija de acuerdo con el tipo de procedimiento endoscópico y a la valoración preanestésica.

\section{SEDACIÓN MÍNIMA (ansiólISIS)}

Es la depresión mínima del estado de consciencia inducida por fármacos, con capacidad del paciente de mantener la vía aérea de forma independiente y continua, sin alteración de la función cardiovascular. Responde de forma adecuada a estimulación física o indicación verbal ${ }^{3,6,13,14}$ (Fig. 1).

\section{SEDACIÓN MOdeRAda (SEDACIÓN CONSCIENTE)}

Es la depresión de la consciencia inducida por fármacos específicos, donde el paciente responde a determinadas órdenes verbales, sin alteración de la función ventilatoria y cardiovascular6,13-15 (Fig. 1).

\section{SEDACIÓN PROFUndA}

Es el estado de depresión del estado de consciencia inducida por fármacos durante la cual los pacientes solo responden después de la estimulación táctil repetida o dolorosa. No hay respuesta a la estimulación verbal y se acompaña de pérdida parcial de los reflejos protectores, incluyendo la incapacidad de mantener la vía aérea de forma independiente y continua. La función cardiovascular generalmente está conserva$\mathrm{da}^{3,6,13,14}$ (Fig. 1).

\section{Anestesia general}

Es el estado controlado de inconsciencia acompañado de la pérdida parcial o total de los reflejos protectores, incluyendo la incapacidad de mantener la vía aérea de forma independiente y continua y/o responder a estimulación física o verbal. Los pacientes requieren asistencia para mantener la vía aérea y la ventilación $n^{3,6,13,14}$ (Fig. 1). 


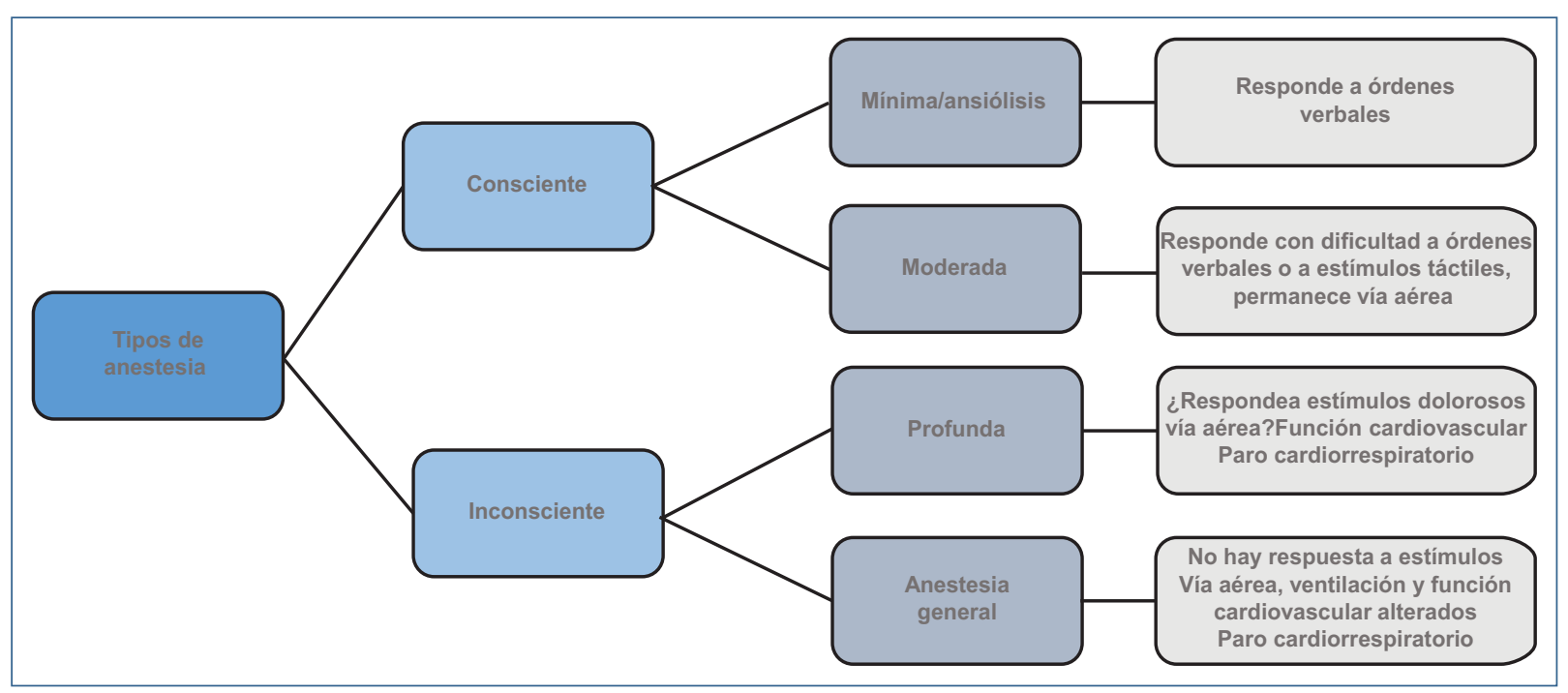

Figura 1. Tipos de anestesia.

\section{Procedimientos anestésicos en endoscopia diagnóstica y terapéutica}

La AMEG recomienda sedación en las colonoscopias para alcanzar éxito técnico del procedimiento y mejorar la satisfacción del paciente.

La AMEG no considera mandatoria la sedación durante la rectosigmoidoscopia, así como en algunos casos de las EGD diagnósticas.

La endoscopia sin sedación tiene ventajas potenciales como menor costo, mayor disponibilidad, evita los riesgos inherentes a la sedación, permite una recuperación más rápida después del procedimiento y no requiere un acompañante, lo que permite a los pacientes conducir o volver a trabajar posteriormente ${ }^{15}$.

Para favorecer la realización de EGD sin sedación se han utilizado endoscopios ultradelgados de introducción transnasal, así como técnicas de inmersión en agua que permitan una colonoscopia sin sedación ${ }^{16,17}$. En un metaanálisis Sami, et al. demostraron que el uso de endoscopios ultradelgados (<5.9 mm) vía transnasal o transoral es una alternativa aceptable con tasas de éxito similares a la endoscopia convencional (intervalo de confianza del 95\% [IC 95\%]: -3.0 a 0) en pacientes a los que se realiza con fines de escrutinio ${ }^{16}$. En un estudio aleatorizado donde se comparó el uso de inmersión en agua vs. la insuflación de aire en paciente a los que se realizó colonoscopia sin sedación mostró una mayor tasa de intubación cecal con el uso de agua (78 vs. $98 \%$ ), así como el deseo de repetirse la colonoscopia $(78 \text { vs. } 93 \%)^{18}$.
La sedación en colonoscopia permite incrementar la tasa de intubación cecal, la tasa de detección de pólipos (razón de momios [RM]: 1.172; IC 95\%: 1.0741.286) y la finalización del estudio ${ }^{9}$, lo que incrementa la satisfacción del paciente y del personal involucrado, y aumenta la probabilidad de que el paciente esté dispuesto a repetir la evaluación endoscópica ${ }^{7,9,19}$.

En un estudio aleatorizado doble ciego, la sedación fue el principal determinante de una endoscopia exitosa, definida como técnicamente adecuada y satisfactoria tanto para el endoscopista como para el paciente (RM: 3.8; IC 95\%: 2.5-5.7), pero tuvo un costo económico mayor y requirió más tiempo de recuperación (29 vs. 15 minutos; $p<0.0001$ ). Dichas diferencias no fueron evidentes en sujetos $>75$ años ${ }^{20}$. Resultados similares fueron reportados por un metaanálisis ${ }^{12,21}$. La sedación mejoró la satisfacción del paciente (riesgo relativo [RR]: 2.29; rango: 1.16-4.53) y la disposición a repetir el estudio (RR: 1.25; rango: 1.13-1.38), con un riesgo bajo de eventos adversos graves $(<1 \%)$, especialmente cuando el procedimiento fue asistido por anestesiólogos.

\section{Procedimiento anestésico en procedimientos endoscópicos complejos}

La AMEG considera indispensable la asistencia del anestesiólogo durante procedimientos complejos o prolongados como enteroscopia asistida, ultrasonido endoscópico (USE), colangiopancreatografía retrograda endoscópica (CPRE), resección 
endoscópica de la mucosa (REM) y disección endoscópica de la submucosa (DES), entre otros. El anestesiólogo y el endoscopista elegirán en conjunto el manejo integral del paciente.

En la tabla 1 se muestran los procedimientos endoscópicos que por su dificultad técnica suelen tener tiempos de duración prolongados y una tasa de complicaciones mayor, comparados con otros procedimientos endoscópicos.

La CPRE, por ejemplo, presenta varios retos para el personal involucrado en el procedimiento. El paciente suele colocarse en decúbito prono, se realiza en salas con poca iluminación, son procedimientos prolongados y con frecuencia los pacientes tienen múltiples comorbilidades y cirugías abdominales recientes. La REM, la DES y la toma de biopsias guiadas por USE son procedimientos prolongados que requieren de gran habilidad técnica y evitar movimientos involuntarios del paciente para realizarlos. La enteroscopia asistida, por la naturaleza del intestino delgado, es un procedimiento particularmente prolongado.

Estas características particulares de los procedimientos endoscópicos complejos hacen que requieran una sedación mas profunda y prolongada, además de que algunos casos podrían requerir de intubación orotraqueal (IOT). Esta debe ser considerada en aquellos sujetos con hemorragia de tubo digestivo y/o retención de sangre en el esófago o estómago, por el alto riesgo de broncoaspiración ${ }^{22-24}$ (Tabla 2).

\section{Medicamentos utilizados durante la anestesia y sedación en endoscopia}

Para garantizar la seguridad y comodidad del paciente, el nivel de sedación debe ajustarse a las necesidades individuales considerando el procedimiento que realizar y las comorbilidades.

Un principio clave en la administración de fármacos es utilizar dosis escalonadas hasta conseguir el grado de sedación y analgesia deseado. El primer objetivo de la sedación es cubrir el componente hipnótico o de inconsciencia, para lo cual el fármaco más utilizado en la actualidad es el propofol; el uso de benzodiacepinas se limita cada vez más en pacientes de la tercera edad por su asociación con delirio y deterioro cognitivo $0^{4,6,25}$.

El régimen de sedación clásico generalmente consiste en una benzodiacepina para minimizar la ansiedad. Las benzodiacepinas ocasionan depresión ventilatoria dependiente de la dosis, pero con apneas de menor duración en comparación con propofol. Antes de presentarse la sedación profunda pueden
Tabla 1. Procedimientos endoscópicos complejos

\begin{tabular}{|c|c|}
\hline Procedimiento & Procedimiento detallado \\
\hline \multirow[t]{5}{*}{ EGD } & $\begin{array}{l}\text { Polipectomía difícil/EMR-EMD, ablación por } \\
\text { radiofrecuencia de esófago de Barrett }\end{array}$ \\
\hline & Procedimiento endoscópico bariátrico \\
\hline & Hemorragia variceal \\
\hline & $\begin{array}{l}\text { Extracción de cuerpo extraño, colocación de } \\
\text { prótesis }\end{array}$ \\
\hline & $\begin{array}{l}\text { Procedimientos terapéuticos prolongados }> \\
60 \mathrm{~min}\end{array}$ \\
\hline \multirow{3}{*}{$\begin{array}{l}\text { Páncreas/ } \\
\text { hepatobiliar }\end{array}$} & USE terapéutico \\
\hline & CPRE \\
\hline & Colangioscopia \\
\hline \multirow{3}{*}{$\begin{array}{l}\text { Intestino } \\
\text { delgado }\end{array}$} & Enteroscopia asistida \\
\hline & Polipectomía compleja/EMR-EMD \\
\hline & $\begin{array}{l}\text { Procedimientos terapéuticos prolongados }> \\
60 \text { min }\end{array}$ \\
\hline
\end{tabular}

EGD: esófago-gastro-duodenoscopia; REM-DES: resección endoscópica de la mucosa-disección endoscópica de la submucosa; USE: ultrasonido endoscópico; CPRE: colangio pancreatografía.

Tabla 2. Indicaciones de intubación orotraqueal en procedimientos endoscópicos avanzados*

\begin{tabular}{|l|l|c|}
\hline Parámetro & Condición & Puntuación \\
\hline $\begin{array}{l}\text { Índice de masa } \\
\text { corporal }\end{array}$ & $<25$ & 0 \\
\hline & $25-35$ & 1 \\
\hline $\begin{array}{l}\text { Tiempo de } \\
\text { procedimiento }\end{array}$ & $\begin{array}{l}\text { Electivo } \\
\text { Urgente, fin semana, festivo }\end{array}$ & 2 \\
\hline $\begin{array}{l}\text { Riesgo de } \\
\text { aspiración }\end{array}$ & Ayuno/no ERGE & 0 \\
\hline $\begin{array}{l}\text { Estado cardiaco/ } \\
\text { hemodinámico }\end{array}$ & Estable & 0 \\
\hline $\begin{array}{l}\text { Vía aérea según } \\
\text { escala } \\
\text { Mallampati }\end{array}$ & I-II & 1 \\
\hline
\end{tabular}

*Intubación con cuatro o más puntos o si el paciente tiene estómago lleno u obstrucción del tracto de salida gástrico. Es el anestesiólogo quien decidirá quién requiere intubación orotraqueal de acuerdo con la naturaleza del procedimiento. ERGE: enfermedad por reflujo gastroesofágico.

aparecer episodios de verborrea y desinhibición psicomotriz y en algunos casos con excitación paradójica 4 . Los efectos cardiovasculares son escasos o nulos cuando se administran solas. La más utilizada es el midazolam y se prefiere por su rápido inicio de acción, 
Tabla 3. Medicamentos utilizados durante la anestesia y sedación en endoscopia

\begin{tabular}{|c|c|c|c|c|c|c|c|}
\hline Fármaco & $\begin{array}{l}\text { Inicio } \\
\text { acción }\end{array}$ & Duración & Metabolismo & Excreción & Antídoto & Efecto & $\begin{array}{l}\text { Eventos } \\
\text { adversos }\end{array}$ \\
\hline \multicolumn{8}{|l|}{ Benzodiazepinas } \\
\hline Midazolam & $1-2 \mathrm{~min}$ & $15-80 \mathrm{~min}$ & Hepático & Renal & Flumazenilo & $\begin{array}{l}\text { Sedante, ansiolítico y } \\
\text { amnésico }\end{array}$ & $\begin{array}{l}\text { Depresión } \\
\text { cardiorrespiratoria }\end{array}$ \\
\hline \multicolumn{8}{|l|}{ Opiodes } \\
\hline Fentanilo & $2.3 \mathrm{~min}$ & $30-60 \mathrm{~min}$ & Hepático & Renal & Naloxona & $\begin{array}{l}\text { Analgésico, sedante, } \\
\text { no amnésico }\end{array}$ & $\begin{array}{l}\text { Depresión respiratoria, } \\
\text { tórax leñoso }\end{array}$ \\
\hline \multicolumn{8}{|l|}{ Inductores del sueño } \\
\hline Ketamina & $30-50 \mathrm{~s}$ & $5-20 \mathrm{~min}$ & Hepático & Renal & Ninguno & $\begin{array}{l}\text { Disociativo, sedante, } \\
\text { analgésico, } \\
\text { alucinógeno }\end{array}$ & $\begin{array}{l}\text { Depresión } \\
\text { cardiorrespiratoria } \\
\text { mínima, reflejos } \\
\text { protectores preservados }\end{array}$ \\
\hline Propofol & $30-45 \mathrm{~s}$ & $4-8 \mathrm{~min}$ & Hepático & Renal & Ninguno & $\begin{array}{l}\text { Sedante, amnésico, no } \\
\text { analgésico }\end{array}$ & $\begin{array}{l}\text { Depresión respiratoria, } \\
\text { hipotensión, dolor en sitio } \\
\text { de inyección }\end{array}$ \\
\hline Dexmedetomidina & $5 \min$ & $15 \mathrm{~min}$ & Hepático & Renal & Atipamezol & $\begin{array}{l}\text { Sedante, analgésico, } \\
\text { ahorrador de opioides }\end{array}$ & $\begin{array}{l}\text { Hipotensión, hipertensión } \\
\text { y bradicardia }\end{array}$ \\
\hline
\end{tabular}

corta duración (vida media menor a 6 horas) y altas propiedades amnésicas. El midazolam produce disminución de las cifras de tensión arterial (TA), manteniéndose la frecuencia cardiaca (FC), la presión de llenado y el gasto cardiaco, sobre todo en pacientes que presentan previamente un tono vascular alto (hipertensos, hipovolémicos). Debido a su metabolismo hepático y eliminación renal, la dosis debe disminuirse en estos pacientes, esperando una acción más rápida y de mayor duración que en pacientes sanos ${ }^{4,6,25}$ (Tabla 3).

El propofol es el anestésico intravenoso más utilizado hoy en día. Es un hipnótico con características cinéticas que lo convierten en un fármaco ideal para endoscopia gastrointestinal. Tiene inicio de acción rápido (20 segundos) y vida media corta (4 a 5 minutos). Produce un efecto antiemético y sensación de bienestar después de la administración. Un efecto secundario de vital importancia en endoscopia es la depresión del sistema respiratorio cuya incidencia y duración depende de la dosis, la velocidad de inyección y de la medicación concomitante. La frecuencia de apnea prolongada es mayor si se agregan opioides a altas concentraciones. El efecto cardiovascular más significativo es la disminución de la presión arterial dependiente de la dosis y comorbilidades del paciente 4,6,25,26. $^{\text {. }}$

Los opioides son la piedra angular para mitigar el dolor; además causan sedación, euforia y tolerancia dependiente de la dosis. Producen frecuentemente efectos adversos como depresión respiratoria, náuseas y vómitos (disminuyen el tono del esfínter gastroesofágico y el vaciamiento gástrico). Adicionalmente pueden causar depresión respiratoria, bradicardia y vasodilatación. El opioide más utilizado hoy en día en procedimientos endoscópicos es el fentanilo. Sus efectos analgésicos a dosis bajas como las requeridas en endoscopia duran entre 15 minutos a 30 minutos, con un inicio de acción de 30 segundos y efecto máximo a los 3.6 minutos $^{4}$.

La dexmedetomidina es un agonista $\alpha 2$ adrenérgico con propiedades sedantes, ansiolíticas y analgésicas, por lo que disminuye la necesidad de opioides. Una ventaja importante de la dexmedetomidina sobre los fármacos anteriores es que a dosis clínicamente efectivas no produce alteraciones en la ventilación. La dosis de carga presenta con mucha frecuencia disminución del impulso simpático y del tono vasomotor, con vasodilatación y disminución de la resistencia vascular periférica y de la presión arterial. Este efecto puede ser mucho más significativo y evidente en el paciente gravemente enfermo, en el que se utilizan esquemas de administración diferentes ${ }^{25,27,28}$.

La ketamina es un bloqueador del receptor $N$-metil d-aspartato que participa en la regulación del potencial excitatorio postsináptico, teniendo un rol preponderante en la plasticidad neuronal, el aprendizaje y la memoria. También interactúa además con receptores colinérgicos, noradrenérgicos, dopaminérgicos y serotoninérgicos. Los únicos usos vigentes hoy en día son sus efectos analgésicos y antidepresivos. Proporciona 
mejor estabilidad hemodinámica, menos depresión respiratoria y limita la cantidad de propofol requerido. Puede ocasionar distintos grados de hipertonía, movimientos musculares, alucinaciones y delirio.

El perfil cardiovascular de la ketamina se ha relacionado con varios mecanismos que incluyen la actividad simpática aumentada por estimulación del sistema nervioso autónomo y la inhibición de la captación de catecolaminas intraneuronal y extraneuronal, generando con ello incremento significativo de la presión arterial, frecuencia cardiaca y de la presión de la arteria pulmonar. Algunos de estos efectos desaparecen o disminuyen su intensidad cuando se utilizan dosis subhipnóticas o cuando se administra en perfusión continua ${ }^{28}$.

\section{Valoración previa a endoscopia y procedimiento anestésico}

\section{Consentimiento informado}

La AMEG recomienda la obtención de consentimiento informado del procedimiento anestésico además del endoscópico.

Es derecho de todo paciente recibir la información clara y apropiada sobre el procedimiento anestésico al cual será sometido. Está información debe ser proporcionada por el anestesiólogo que realizará el procedimiento antes de este y debe incluir: la indicación médica, descripción del procedimiento, las diferentes alternativas, los riesgos y beneficios, así como las principales complicaciones. La decisión de realizar el procedimiento se confirma con la firma del paciente del consentimiento informado ${ }^{29,30}$.

El consentimiento informado es el o los documentos escritos y signados por el paciente o su representante legal o familiar más cercano en vínculo, mediante los cuales se acepta la realización de un procedimiento médico o quirúrgico con fines diagnósticos, terapéuticos, rehabilitatorios, paliativos o de investigación, una vez que el paciente y/o su representante ha recibido información sobre los riesgos y beneficios esperados ${ }^{2}$. El consentimiento del paciente es temporal y revocable sin sujeción a formalidad alguna.

La NOM-004-SSA3-2012 establece que el consentimiento informado debe contener los siguientes elementos obligados:

1. Establecimiento médico donde se llevará acabo el procedimiento.

2. Nombre o razón social del establecimiento médico.

3. Titulo del documento.
4. Lugar y fecha.

5. Acto autorizado.

6. Señalamiento de los riesgos y beneficios esperados del acto médico autorizado.

7. Autorización al personal de salud para la atención de contingencias y urgencias derivadas del acto autorizado, atendiendo al principio de libertad prescriptiva.

8. Nombre y firma de la persona que otorga la autorización.

9. Nombre y firma de los testigos (en caso de amputación, mutilación o extirpación orgánica que produzca modificación física permanente 0 en la condición fisiológica o mental del paciente).

10. Nombre completo y firma del que realiza el acto quirúrgico.

Se elaboran tantos consentimientos como eventos médicos ameritan.

\section{Historia clínica y exploración física dirigidas (consulta preanestésica)}

La AMEG recomienda determinar la adecuada preparación del paciente previo al procedimiento anestésico y endoscópico.

La AMEG recomienda que se realice una consulta anestésica que recabe los principales antecedentes médicos y realice una exploración física dirigida a detectar las principales comorbilidades que puedan modificar el procedimiento anestésico y endoscópico.

Todo procedimiento endoscópico requiere de una preparación, cuyo cumplimiento debe ser confirmado previamente. La endoscopia conlleva un riesgo de broncoaspiración, por lo que todo paciente debe cumplir con un tiempo de ayuno ${ }^{31}$ mínimo, el cual no ha sido definido. En la actualidad se sugiere no ingerir líquidos claros o alimentos sólidos durante al menos 3 horas y 6 horas respectivamente previo al procedimiento.

Es importante considerar e identificar pacientes con retraso en el vaciamiento gástrico asociado a alguna patología o bien secundario a fármacos, en cuyo caso el tiempo de ayuno deberá individualizarse.

No se recomienda el uso sistemático de procinéticos, pues su utilidad es limitada. Estos fármacos han sido estudiados en pacientes con hemorragia gastrointestinal alta, donde han demostrado reducir la necesidad de un segundo procedimiento, sin impacto en otros parámetros $^{32}$. La intubación profiláctica en pacientes con hemorragia digestiva alta debe individualizarse ${ }^{33,34}$.

Se deben consignar antecedentes médicos como alergias y comorbilidades con especial atención a enfermedades cardiopulmonares, que incrementan el 
riesgo de complicaciones asociadas a la sedación, como arritmias e hipoxemia. Debe documentarse el antecedente de efectos adversos relacionados. Las alteraciones del funcionamiento renal y hepático interfieren con el metabolismo y excreción de los fármacos empleados durante el procedimiento ${ }^{35}$. La obesidad, edad avanzada, embarazo y población pediátrica son condiciones que requieren especial atención ${ }^{36}$. El examen físico antes de los procedimientos anestésicos debe evaluar signos vitales, nivel del estado de alerta y predictores para vía aérea difícil que se comentarán más adelante.

\section{Clasificación del riesgo del paciente}

La AMEG recomienda realizar previo al procedimiento endoscópico una evaluación dirigida a la detección de predictores de riesgo asociados al acto anestésico.

La evaluación del riesgo anestésico-quirúrgico en un paciente que será intervenido quirúrgicamente debe considerar la edad, estado cardiovascular y función respiratoria. Con el objetivo de facilitar y estandarizar este proceso se han desarrollado diferentes escalas que clasifican el riesgo de acuerdo con el estado físico del paciente (Sociedad Americana de Anestesiólogos [ASA]) y su funcionalidad (Asociación del Corazón de Nueva York), entre otras ${ }^{37,38}$.

\section{EDAD}

Debe considerarse que la mortalidad se incrementa en sujetos mayores de 65 años y se incrementa proporcionalmente con la complejidad del procedimiento y la presencia de comorbilidades.

\section{ESTADO CARDIOVASCULAR}

Es el predictor más importante de mortalidad operatoria. Factores como enfermedad valvular, reoperación, función ventricular izquierda, infarto de miocardio reciente (menos de 12 semanas), insuficiencia cardiaca, cirugía de urgencia y trastornos del ritmo se asocian a complicaciones postoperatorias 39,40 .

\section{FUNCIÓN RESPIRATORIA}

La enfermedad pulmonar obstructiva crónica es un factor de riesgo asociado a ventilación mecánica prolongada.

\section{Vía AÉREA}

La naturaleza de los procedimientos endoscópicos, especialmente aquellos del tubo digestivo alto, requieren establecer y conocer las indicaciones y cuidados necesarios para una adecuada monitorización y protección de la vía aérea, considerando la indicación del procedimiento, así como la facilidad o dificultad que esta ofrece para su acceso e intubación.

Es importante identificar una vía aérea difícil de manera oportuna, pues esto evitará complicaciones y situaciones apremiantes que compliquen y pongan en peligro la integridad y vida del paciente. Para esto se desarrollaron predictores de vía aérea difícil que incluyen las escalas de: Mallampati, Patil-Aldreti, la distancia interincisivos y la escala atlantooccipital ${ }^{41}$.

Independientemente de la indicación y urgencia del procedimiento, tanto el endoscopista como el anestesiólogo deben tener la capacidad de detectar aquellas situaciones médicas o extramédicas que lleven a cancelar o diferir el procedimiento, incluyendo el no contar con la infraestructura adecuada (NOM-016-SSA3-2012)².

\section{Tiempo fuera y lista de verificación}

\section{TIEMPO FUERA}

La AMEG sugiere realizar una pausa (tiempo fuera) previo al inicio del procedimiento endoscópico y anestésico durante la cual se revise la lista de verificación (LV) que permita confirmar la identidad y consignar aspectos clínicos y paraclínicos relevantes.

En cirugía, con el objetivo de identificar y vigilar aspectos clínicos y paraclínicos importantes previo y posterior al procedimiento quirúrgico, se desarrolló una LV. Esta se revisa previo al procedimiento quirúrgico mediante una pausa llamada tiempo fuera. Durante esta pausa se identifica al paciente, se confirma el procedimiento a realizar, así como el sitio a intervenir y el personal que lo realizará. También se consignan antecedentes médicos de importancia (p. ej., alergias). La aplicación del tiempo fuera ha demostrado disminuir la tasa de mortalidad (del 1.5 al $0.8 \% ; p<0.003$ ) y de complicaciones (del 9 al 7\%; $p<0.001)^{42}$.

En endoscopia el tiempo fuera es el periodo o pausa que ocurre antes del inicio del procedimiento anestésico. El paciente junto con el endoscopista y anestesiólogo confirman la información consignada en la LV.

No existe una LV estandarizada para endoscopia ni estudios que muestren el impacto en la morbimortalidad. Existen listas con diversos contenidos y 
Tabla 4. Lista de verificación en endoscopia Lista de verificación endoscópica

\begin{tabular}{|c|c|c|c|}
\hline \multirow{10}{*}{$\begin{array}{l}\text { Tiempo fuera } \\
\text { (antes de la inserción } \\
\text { del endoscopio y } \\
\text { antes de iniciar el } \\
\text { procedimiento } \\
\text { anestésico) }\end{array}$} & $\begin{array}{l}\text { Confirmar identidad del } \\
\text { paciente (doble } \\
\text { identificador) }\end{array}$ & 0 Sí & \\
\hline & $\begin{array}{l}\text { Presentación del equipo } \\
\text { médico }\end{array}$ & 0 Sí & \\
\hline & Procedimiento correcto & O Sí & \\
\hline & Indicación correcta & O Sí & \\
\hline & $\begin{array}{l}\text { Consentimiento informado } \\
\text { endoscópico }\end{array}$ & 0 Sí & \\
\hline & $\begin{array}{l}\text { Consentimiento } \\
\text { anestésico }\end{array}$ & 0 Sí & \\
\hline & $\begin{array}{l}\text { Equipo y accesorios } \\
\text { completos y funcionales }\end{array}$ & 0 Sí & \\
\hline & $\begin{array}{l}\text { Alergias } \\
\text { (especificar) }\end{array}$ & 0 Sí & $0 \mathrm{No}$ \\
\hline & Antitrombóticos & 0 Sí & $0 \mathrm{No}$ \\
\hline & $\begin{array}{l}\text { Se retiró prótesis dental } \\
\text { (en caso de que la } \\
\text { utilice) }\end{array}$ & O Sí & $\mathrm{O}$ No \\
\hline
\end{tabular}

Adaptada de Matharoo, et al., $2014^{46}$.

sugerimos que esta contenga: identidad del paciente mediante doble verificador (p. ej., nombre y fecha nacimiento, edad), presentación de cada integrante del equipo de endoscopia, indicación del estudio, firma de los consentimientos informados, uso de prótesis dentales, comorbilidades importantes, alergias, uso de antitrombóticos, correcto funcionamiento del equipo y monitorización adecuada ${ }^{43,44}$ (Tabla 4).

\section{Monitoreo y cuidados transprocedimiento}

La hipoxia (saturación de oxígeno medida con oxímetro de pulso $\left.\left[\mathrm{SpO}_{2}\right]<90 \%\right)$ es el evento adverso más común durante los procedimientos anestésicos y endoscópicos. La hipoxia severa $\left(\mathrm{SpO}_{2}<85 \%\right)$ a menudo requiere detener temporalmente el procedimiento endoscópico para dar ventilación con mascarilla o incluso la IOT. La hipoxia prolongada puede causar isquemia miocárdica, arritmia cardiaca, daño neurológico permanente 0 incluso la muerte ${ }^{45}$.

Por este motivo se utilizan métodos para reducir la incidencia de hipoxia durante el procedimiento anestésico. Bell, et al. demostraron que aportar oxígeno con una cánula nasal durante el procedimiento redujo la incidencia de hipoxia del 77 al $16 \%{ }^{46}$.

\section{Utilidad del monitoreo de oximetría de pulso y capnografía transprocedimiento}

La AMEG considera indispensable el uso de oximetría de pulso en todos los pacientes que sean sometidos a procedimientos anestésicos para endoscopia.

En los pacientes sometidos a sedación profunda se recomienda considerar el uso de capnografía que permite detectar de manera temprana alteraciones respiratorias (hipoventilación), previo a la aparición de hipoxia.

La Norma Oficial Mexicana NOM-006-SSA3-2011 establece que en todo procedimiento anestésico, el médico anestesiólogo debe vigilar al paciente apoyado por la oximetría de pulso. La monitorización convencional de los pacientes durante la endoscopia, recomendada por las guías clínicas de la Sociedad Americana de Endoscopia Gastrointestinal (ASGE), la Sociedad Europea de Endoscopia Gastrointestinal (ESGE) y la Sociedad Americana de Anestesiólogos (ASA) establecen la vigilancia automática no invasiva de la TA, FC, saturación de oxígeno $\left(\mathrm{SatO}_{2}\right)$ por oximetría de pulso continua y valoración visual de la actividad ventilatoria (permeabilidad de la vía aérea, frecuencia y profundidad de la respiración), así como del nivel del estado de consciencia del paciente. La oximetría de pulso se considera necesaria para cualquier endoscopia bajo procedimiento anestésico ${ }^{47,48}$. Algunos de los factores de riesgo para hipoxemia en estos pacientes son: $\mathrm{SatO}_{2}$ basal $<95 \%$, dificultad para lograr intubación esofágica por el endoscopista, presencia de comorbilidades, endoscopia urgente y mayor duración del procedimiento ${ }^{47}$.

La capnografía y capnometría son recomendables en todo procedimiento de anestesia general ${ }^{2}$. La vigilancia visual de la actividad respiratoria, particularmente en procedimientos endoscópicos prolongados, no es un método confiable para la detección de apnea. El capnógrafo mide el dióxido de carbono al final de la espiración, con lo que se logra detectar de manera temprana la depresión respiratoria antes de que se manifieste la hipoxia ${ }^{48}$.

El uso de la capnografía como parte de los protocolos de monitorización en sedación moderada es controvertido ${ }^{47}$. Diversos ensayos clínicos aleatorizados, metaanálisis y revisiones sistemáticas han demostrado que la capnografía disminuye el número de episodios de apnea y reduce la tasa de hipoxemia (RR: 0.58; IC 95\%: $0.26-1.27 ; p=0.17)^{49}$, tanto de manera global (RM: 0.61; IC 95\%: 0.49-0.77) como los casos de hi-

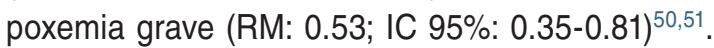


Dichos estudios no muestran impacto en eventos clínicamente significativos como bradicardia, hipotensión, requerimiento de oxígeno suplementario o manejo invasivo de la vía aérea, modificación en la dosis de sedantes, tasa de procedimientos incompletos o satisfacción del paciente ${ }^{49-51}$.

La capnografía ha demostrado utilidad en pacientes sometidos a sedación profunda, procedimientos endoscópicos prolongados o cuando no es posible una vigilancia visual apropiada de la respiración del paciente. Debido a que no ha demostrado un impacto clínico relevante, no se puede recomendar su uso cotidiano y/o generalizado.

\section{El índice biespectral ayuda a determinar la profundidad de la sedación}

El electroencefalograma es la vía mas útil para la investigación de la actividad cerebral, pues nos muestra claras modificaciones en la actividad cerebral cortical durante la sedación, sueño profundo y anestesia general, complementando de manera fácil la valoración clínica y viceversa.

El índice biespectral (BIS) es una monitorización que se ha usado para visualizar numéricamente la actividad cortical cerebral; los rangos oscilan entre 0 y 100 (0, coma; 40 a 60 anestesia o sueño profundo; 60 a 90 sedación o somnolencia; 90 a 100 despierto), aunque se debe considerar que esto puede variar dependiendo del estado clínico del paciente y del tipo de fármaco que se use para anestesia ${ }^{52,53}$.

El uso de BIS reduce hasta un $19 \%$ el requerimiento de anestésicos en comparación con una monitorización estándar; mejora el tiempo de recuperación de una anestesia profunda y reduce la incidencia de riesgo de despertar intraoperatorio y el reingreso del paciente a nuevos procedimientos quirúrgicos ${ }^{54}$.

La utilidad y beneficios del BIS no han sido evaluados en endoscopia, por lo que no es posible emitir alguna recomendación respecto a su uso o papel en estos procedimientos.

\section{Utilidad de la terapia de oxígeno de alto flujo transprocedimiento}

La AMEG considera que el uso de terapia de oxígeno de alto flujo (OAF) no es necesario de manera sistemática en pacientes sometidos a procedimiento anestésico en endoscopia, pero puede considerarse en casos seleccionados.
La OAF con cánula nasal es un sistema que permite administrar mezclas cálidas y humidificadas de oxígeno y aire a flujos de hasta $60 \mathrm{l} / \mathrm{min}$. La concentración de oxígeno inspirado puede variar de 21 al $100 \%{ }^{55}$. Los efectos fisiológicos de esta terapia son: lavado del espacio muerto nasofaríngeo, reducción del trabajo respiratorio, mantenimiento del efecto de presión positiva al final de la espiración y mantenimiento de la función mucociliar ${ }^{55,56}$.

Las principales áreas donde existe evidencia sobre la utilidad de la OAF es en adultos con insuficiencia respiratoria aguda y crónica, poscirugía cardíaca, insuficiencia cardiaca, preintubación, postextubación y procedimientos invasivos (p. ej., los endoscópicos) ${ }^{57}$.

En endoscopia, la OAF comparando con oxígeno por cánula nasal (puntas nasales) disminuyó significativamente los periodos de desaturación de oxigeno capilar ${ }^{58}$.

En procedimientos endoscópicos bajo sedación profunda (p. ej., CPRE y USE) la saturación en casos recibiendo OAF fue similar a la registrada bajo anestesia general, reduciendo significativamente el tiempo total del procedimiento ${ }^{59}$.

También en endoscopia superior electiva bajo sedación con propofol la OAF comparada con puntas nasales ha demostrado reducir los periodos de hipoxia del 8.4 al $0 \%(p<0.001)$ e hipoxia severa del 0.6 al $0 \%(p=0.03)^{60}$.

Un estudio mexicano reportó resultados similares que favorecen el uso de OAF sobre las puntas nasales en procedimientos endoscópicos bajo sedación, al disminuir los episodios de desaturación ( $p$ $=0.009)^{61}$.

En pacientes con obesidad mórbida en colonoscopia no se encontraron diferencias en el número de episodios de desaturación comparando OAF con la cánula nasal convencional (39 vs. $45 \%$ ), lo que posiblemente esté relacionado con los cambios anatómicos en la orofaringe e hipofaringe observados en estos pacientes y que se asocian con obstrucción parcial de la vía aérea superior ${ }^{62}$.

La OAF ofrece beneficios sobre las puntas nasales en casos seleccionados.

Cuando ocurra un evento de desaturación de oxígeno durante un procedimiento endoscópico se recomienda el algoritmo mostrado en la figura 2. 


\section{Cuidados posprocedimiento}

\section{Monitorización del paciente al término de la exploración endoscópica}

La AMEG considera que toda unidad de endoscopia debe contar con personal capacitado para evaluar el estado clínico del paciente durante su recuperación y un protocolo de egreso (se recomienda utilizar la escala de Aldrete). El paciente deberá recibir información específica respecto a cuidados posanestésicos al momento de su egreso.

La monitorización del paciente en el periodo posprocedimiento permite la detección de complicaciones de manera temprana previo al egreso del paciente y determinar el momento en el cual el paciente cumple con criterios que permitan su egreso.

La ASGE considera como un criterio de calidad en endoscopia el contar con parámetros establecidos de egreso de pacientes, que deberán documentarse en el reporte endoscópico (meta de documentación > 98\% de los casos) $)^{63}$.

Durante la monitorización posprocedimiento $0^{4,64-66}$ :

- Debe existir personal capacitado asignado al área de recuperación para vigilar al paciente, así como para reconocer y tratar las complicaciones derivadas de la anestesia y/o sedación.

- Debe monitorizarse al paciente inmediatamente después del procedimiento endoscópico y hasta antes del alta. Se tiene que vigilar la ventilación cada 5-15 minutos (dependiendo de las condiciones particulares de cada paciente) hasta que recupere su estado de alerta basal.

- La evaluación del paciente debe incluir la valoración de su función neuromuscular y neurológica, identificando potenciales complicaciones con el fin de reducir eventos adversos. Para este fin deben utilizarse escalas validadas (p. ej., la de Aldrete).

- Se deben establecer criterios de alta para minimizar el riesgo de depresión de sistema nervioso central y/o cardiorrespiratorio.

- Cuando se utilizan antagonistas de los sedantes la vigilancia deberá ser más prolongada.

\section{Durante el cuidado posprocedimiento, la escala de Aldrete es útil para valorar los signos postanestésicos}

La AMEG recomienda el uso de la escala de Aldrete en la evaluación clínica posprocedimiento para decidir el egreso del paciente sometido a procedimiento anestésico en endoscopia.
Tabla 5. Escala de Aldrete para procedimientos

\begin{tabular}{|c|c|c|}
\hline Variable & Hallazgos & Puntuación \\
\hline Actividad & $\begin{array}{l}\text { Mueve voluntariamente } 0 \text { ante } \\
\text { órdenes } 4 \text { extremidades } \\
\text { Mueve voluntariamente } 0 \text { ante } \\
\text { órdenes } 2 \text { extremidades } \\
\text { Incapaz de mover extremidades }\end{array}$ & $\begin{array}{l}2 \\
1 \\
0\end{array}$ \\
\hline Respiración & $\begin{array}{l}\text { Capaz de respirar profundamente } \\
\text { y toser libremente } \\
\text { Disnea o respiración limitada } \\
\text { Apnea }\end{array}$ & $\begin{array}{l}2 \\
1 \\
0\end{array}$ \\
\hline Circulación & $\begin{array}{l}\text { Tensión arterial < 20\% del nivel } \\
\text { presedación } \\
\text { Tensión arterial } 20-49 \% \text { del nivel } \\
\text { presedación } \\
\text { Tensión arterial > 50\% del nivel } \\
\text { presedación }\end{array}$ & $\begin{array}{l}2 \\
1 \\
0\end{array}$ \\
\hline Consciencia & $\begin{array}{l}\text { Completamente despierto } \\
\text { Responde a estímulos verbales } \\
\text { No responde }\end{array}$ & $\begin{array}{l}2 \\
1 \\
0\end{array}$ \\
\hline Saturación & $\begin{array}{l}\text { Saturación }>95 \% \text { al aire ambiente } \\
\text { Necesita oxígeno para mantener } \\
\text { saturación }>90 \% \\
\text { Saturación }<90 \% \text { con oxígeno }\end{array}$ & $\begin{array}{l}2 \\
1 \\
0\end{array}$ \\
\hline
\end{tabular}

Todo paciente que haya sido sometido a sedación para procedimiento endoscópico debe ser vigilado hasta su recuperación plena para poder ser egresado. Se recomienda utilizar escalas de puntuación que midan y documenten la recuperación postanestésica y permitan determinar el momento idóneo del alta del paciente. La escala de Aldrete (Tabla 5) es la más utilizada en la práctica clínica. Una puntuación mayor a 9 permite el alta del paciente ${ }^{4}$.

\section{Vigilancia y detección de complicaciones asociadas con el procedimiento anestésico}

La AMEG recomienda que la monitorización de los pacientes se realice por personal médico certificado, capacitado en la detección y manejo oportuno de las complicaciones.

Para una detección oportuna y un manejo efectivo de complicaciones posprocedimiento es indispensable disponer de una sala de recuperación dotada de personal de enfermería capacitado en procedimientos endoscópicos, camillas, oxígeno, aspiración, instrumentos de monitorización y material de soporte cardiopulmonar, así como personal capacitado en reanimación cardiopulmonar ${ }^{4}$. Algunas de las complicaciones relacionadas con el procedimiento anestésico se muestran en la tabla 6 4,65-67. 


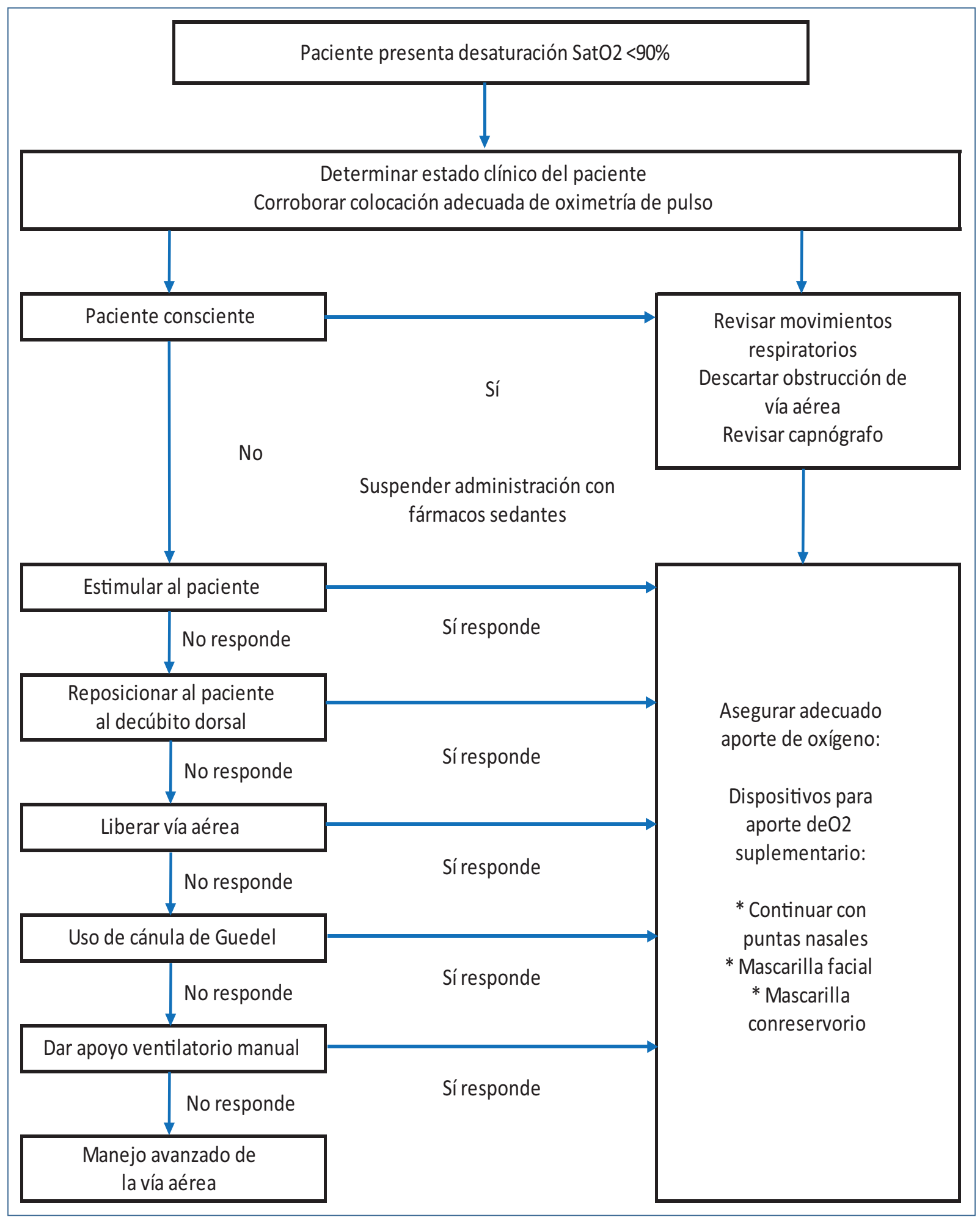

Figura 2. Acciones en caso de desaturación de oxígeno durante el procedimiento endoscópico. Algoritmo basado en la guía de la Sociedad Americana de Anestesiólogos (ASA) para manejo de la vía aérea difícil y el algoritmo ASA-DAS. $\mathrm{SatO}_{2}$ : saturación de oxígeno. 
Tabla 6. Complicaciones asociadas con el procedimiento anestésico

\begin{tabular}{|c|c|c|}
\hline Complicación & Generalidades & Sugerencia \\
\hline Flebitis & $\begin{array}{l}\text { - Por uso de catéter de bajo calibre } \\
\text { - Algunos fármacos provocan irritación de la pared venosa y } \\
\text { provocan dolor }\end{array}$ & $\begin{array}{l}\text { - La lidocaína IV disminuye el dolor local } \\
\text { - Aplicar frío local si hay dolor o datos de flebitis }\end{array}$ \\
\hline $\begin{array}{l}\text { Hipotensión } \\
\text { (PAS }<90 \mathrm{mmHg} \text { ) }\end{array}$ & $\begin{array}{l}\text { - Se asocia con administración de sedantes y analgésicos o } \\
\text { con el uso del propofol } \\
\text { - No suele tener repercusión clínica }\end{array}$ & - Infusión de soluciones parenterales \\
\hline $\begin{array}{l}\text { Hipoxemia } \\
\left(\mathrm{SatO}_{2}<90 \%\right)\end{array}$ & $\begin{array}{l}\text { - Incidencia variable }(4-50 \%) \\
\text { - La administración combinada de benzodiazepinas y opiáceos } \\
\text { aumenta el riesgo de depresión respiratoria }\end{array}$ & - Oxígeno suplementario y asegurar la vía aérea \\
\hline Arritmias & $\begin{array}{l}\text { - Se pueden presentar hasta en el } 4-72 \% \text {. La mayoría son } \\
\text { taquicardias sinusales relacionadas con estímulos propios } \\
\text { del procedimiento }\end{array}$ & $\begin{array}{l}\text { - Monitoreo electrocardiográfico } \\
\text { - En bradicardia (<50 por minuto): administrar } \\
\text { atropina ( } 0.5 \mathrm{mg} \text { IV hasta un máximo de } 2-3 \mathrm{mg} \text { ) }\end{array}$ \\
\hline Broncoaspiración & $\begin{array}{l}\text { - Se presenta en el } 0.10 \% \\
\text { - Más frecuente en hemorragia digestiva alta activa, } \\
\text { gastroparesia u obstrucción al tracto de salida gástrico }\end{array}$ & $\begin{array}{l}\text { - Está recomendada la intubación endotraqueal } \\
\text { antes de realizar la exploración endoscópica }\end{array}$ \\
\hline $\begin{array}{l}\text { Contaminación o } \\
\text { infección }\end{array}$ & - Por uso de envases multidosis y contaminación del fármaco & $\begin{array}{l}\text { - No deben utilizarse envases multidosis para } \\
\text { más de un paciente }\end{array}$ \\
\hline
\end{tabular}

PAS: Presión arterial sistólica; $\mathrm{Sat}_{2}$ : saturación de oxígeno.

\section{Procedimientos anestésicos en casos especiales: pacientes pediátricos, pacientes embarazadas y otros}

\section{Cuidados preprocedimiento, transprocedimiento y posprocedimiento en pacientes pediátricos}

\section{AYUNO PREVIO A ENDOSCOPIA Y PROCEDIMIENTO ANESTÉSICO}

La AMEG recomienda en pacientes pediátricos ayuno no mayor de 8 horas. El tiempo de este dependerá del tipo de alimento consumido. En procedimientos de urgencia se debe considerar que todo paciente tiene el estómago lleno y esto no contraindica el procedimiento endoscópico.

El ayuno preoperatorio se define como el periodo de tiempo entre la última ingesta de alimentos y el inicio del procedimiento. Durante este tiempo, los pacientes no tienen permitida la ingesta oral de líquidos y/o sólidos $^{68}$. Su propósito es reducir la incidencia de regurgitación de contenido gástrico hacia la vía respiratoria (broncoaspiración) ${ }^{69}$ (Tablas 7 y 8).

La broncoaspiración es un evento raro en niños, con una incidencia estimada del $0.07 \%-0.1 \%{ }^{69,70}$, o bien se ha reportado que ocurre en 3 de cada 10,000 ca$\operatorname{sos}^{70,71}$. Estudios de resonancia magnética muestran que el vaciamiento gástrico en niños es rápido para
Tabla 7. Volumen de líquidos claros permitidos por edad

\begin{tabular}{|c|c|c|}
\hline Edad & & Volumen permitido \\
\hline 1-5 años & \multirow{3}{*}{$\begin{array}{l}\text { Agua, jugos sin pulpa, } \\
\text { bebidas diluidas listas, } \\
\text { bebidas deportivas no } \\
\text { gaseosas, líquidos no } \\
\text { espesos, té y café negro } \\
\text { (Tohda, et al., 2006'; NOM-006- } \\
\text { SSA3-20112; ASGE, 20183) }\end{array}$} & Hasta $55 \mathrm{ml}^{2}$ \\
\hline 6-12 años & & Hasta $140 \mathrm{~m}^{2}$ \\
\hline $\begin{array}{l}\text { Mayores de } \\
12 \text { años }\end{array}$ & & Hasta $250 \mathrm{ml}^{2}$ \\
\hline
\end{tabular}

Volúmenes recomendados en caso de no contar con peso exacto o aproximado, y no tener a la mano la cinta de emergencias pediátricas Broselow (cinta Broselof). Adaptada de Andersson, et al., 2018 ${ }^{73}$.

líquidos claros (30 minutos) $)^{71}$, los cuales se eliminan del estómago a un ritmo de $1 \mathrm{ml} / \mathrm{kg}$ después de 1 hora ${ }^{69}$. Reportes de ultrasonido en niños indican que $10-15 \mathrm{ml} / \mathrm{kg}$ de líquidos claros administrados 2 horas antes de la operación resulta en una reducción en el volumen antral al momento de la inducción ${ }^{70}$.

\section{Premedicación en pacientes pediátricos}

La AMEG recomienda antes de la endoscopia en niños la premedicación con midazolam oral para disminuir la ansiedad en procedimientos ambulatorios y considerar la dexmedetomidina o el midazolam oral en pacientes hospitalizados. 
Tabla 8. Indicaciones de ayuno para procedimientos electivos que requieran anestesia general o sedación

\begin{tabular}{|c|c|c|c|c|c|}
\hline Edad & Tiempo & $\begin{array}{l}\text { Tipo } \\
\text { Alimento }\end{array}$ & $\begin{array}{l}\text { Características de los } \\
\text { alimentos }\end{array}$ & Volumen & Contraindicaciones \\
\hline $\begin{array}{l}\text { 0-16 años } \\
\text { (Andersson, et al., } \\
2018^{73} \text { ) }\end{array}$ & 1 hora & $\begin{array}{l}\text { Líquidos } \\
\text { claros }\end{array}$ & $\begin{array}{l}\text { Agua, jugos sin pulpa, } \\
\text { bebidas diluidas listas, } \\
\text { bebidas deportivas no } \\
\text { gaseosas, líquidos no } \\
\text { espesos, té y café negro }\end{array}$ & Máximo 3 ml/kg & $\begin{array}{l}\text { Reflujo gastroesofágico } \\
\text { (ya sea en tratamiento o en } \\
\text { investigación), insuficiencia } \\
\text { renal, algunas enteropatías, } \\
\text { estenosis esofágicas, } \\
\text { parálisis cerebral severa, } \\
\text { acalasia, diabetes mellitus } \\
\text { con gastroparesia y/o } \\
\text { contraindicaciones } \\
\text { quirúrgicas }\end{array}$ \\
\hline $\begin{array}{l}\text { Recién nacidos } \\
\text { sanos (<44 sem de } \\
\text { gestación) y } \\
\text { lactantes }\end{array}$ & $\begin{array}{l}4 \text { horas } \\
\text { posterior a } \\
\text { terminar la } \\
\text { ingesta }\end{array}$ & $\begin{array}{l}\text { Leche } \\
\text { materna }\end{array}$ & Leche materna & $\begin{array}{l}\text { Volumen } \\
\text { indistinto, el tipo } \\
\text { de alimento tiene } \\
\text { mayor } \\
\text { importancia }\end{array}$ & Ayuno absoluto \\
\hline $\begin{array}{l}\text { Recién nacidos y } \\
\text { lactantes }\end{array}$ & $\begin{array}{l}6 \text { horas } \\
\text { posterior a } \\
\text { terminar la } \\
\text { ingesta }\end{array}$ & $\begin{array}{l}\text { Fórmula } \\
\text { infantil }\end{array}$ & $\begin{array}{l}\text { Fórmula infantil (se } \\
\text { consideran como sólido) } \\
\text { Pueden prologar el tiempo } \\
\text { de vaciado gástrico }\end{array}$ & $\begin{array}{l}\text { Considerar la } \\
\text { cantidad ingerida } \\
\text { al determinar un } \\
\text { periodo de ayuno } \\
\text { apropiado }\end{array}$ & Ayuno absoluto \\
\hline $0-16$ años & 6 horas & $\begin{array}{l}\text { Leche no } \\
\text { humana }\end{array}$ & $\begin{array}{l}\text { Leche de vaca, cabra y } \\
\text { oveja (se consideran } \\
\text { como sólido) } \\
\text { Pueden prologar el tiempo } \\
\text { de vaciado gástrico }\end{array}$ & $\begin{array}{l}\text { Considerar la } \\
\text { cantidad ingerida } \\
\text { al determinar un } \\
\text { periodo de ayuno } \\
\text { apropiado }\end{array}$ & Ayuno absoluto \\
\hline $0-16$ años & $\begin{array}{l}6 \text { horas hasta } 8 \\
\text { horas }\end{array}$ & $\begin{array}{l}\text { Comida } \\
\text { ligera }\end{array}$ & $\begin{array}{l}\text { Incluye alimentos no fritos } \\
\text { y sin grasas (p. ej., } \\
\text { tostadas y líquidos claros) }\end{array}$ & $\begin{array}{l}\text { Volumen } \\
\text { indistinto, el tipo } \\
\text { de alimento tiene } \\
\text { mayor } \\
\text { importancia }\end{array}$ & Ayuno absoluto \\
\hline 0-16 años & 8 horas & $\begin{array}{l}\text { Comida } \\
\text { con grasas }\end{array}$ & $\begin{array}{l}\text { Incluye carnes y } \\
\text { alimentos fritos y con } \\
\text { grasas } \\
\text { Pueden prologar el tiempo } \\
\text { de vaciado gástrico }\end{array}$ & $\begin{array}{l}\text { Considerar la } \\
\text { cantidad ingerida } \\
\text { al determinar un } \\
\text { periodo de ayuno } \\
\text { apropiado }\end{array}$ & Ayuno absoluto \\
\hline
\end{tabular}

La prevalencia reportada de ansiedad en sala de espera en niños de entre 4 y 8 años es del 38.9 al $84 \%{ }^{73}$. La administración de un sedante antes de pasarlo a sala reduce el sufrimiento y facilita la inducción de la anestesia ${ }^{4,74}$.

Los mayores beneficios se han observado en preescolares y adolescentes, pacientes con experiencias hospitalarias previas desagradables y aquellos con dificultades de comunicación o cooperación.

Por su facilidad de administración, seguridad y buena biodisponibilidad por vía oral, el midazolam es la premedicación más utilizada en los niños ${ }^{75,76}$. Otra alternativa es la dexmedetomidina que, como se mencionó previamente, posee propiedades sedantes, ansiolíticas y analgésicas, así como un importante efecto simpaticolítico. Cuando esta se administra por vía oral, su biodisponibilidad es del $81.8 \%$. La administración de dexmedetomidina intranasal comparada con midazolam por vía oral es más eficaz y disminuye la ansiedad preoperatoria en un $28 \%{ }^{77}$.

\section{Se RECOMIENDA LA ADMINISTRACIÓN DE OXíGENO EN TODOS LOS PROCEDIMIENTOS ENDOSCÓPICOS EN PACIENTES PEDIÁTRICOS}

La AMEG considera que en todos los pacientes pediátricos a los que se les realizará algún procedimiento endoscópico el uso de oxígeno es obligatorio. El uso de OAF no es necesario de manera sistemática, pero puede considerarse en casos seleccionados. 
En pediatría los procedimientos endoscópicos se realizan bajo la modalidad de procedimiento anestésico con oxígeno por puntas nasales. Existe correlación entre la técnica de administración de oxígeno con el índice de desaturación (convencional vs. alto flujo). Como se mencionó previamente, el uso de OAF disminuye la probabilidad de hipoxemia, que es uno de los eventos adversos más frecuentes y peligrosos durante la endoscopia bajo sedación $n^{61,78-80}$.

Al igual que en adultos, se recomienda en todos los casos administrar oxígeno (mediante puntas nasales $u$ $\mathrm{OAF}$ ) y monitorizar la $\mathrm{SatO}_{2}$ de manera no invasiva mediante pulsioximetría, frecuencia respiratoria, FC y, de ser posible, capnografía ${ }^{80}$.

\section{SELECCIÓN DEL TIPO DE PROCEDIMIENTO ANESTÉSICO EN PACIENTES PEDIÁTRICOS}

La AMEG recomienda que el tipo procedimiento anestésico en pacientes pediátricos se establezca con base en la edad y el peso del paciente, así como el procedimiento que realizar.

La técnica anestésica dependerá del procedimiento, tipo de paciente y habilidad del endoscopista, así como de las capacidades y limitaciones del área de endoscopia. Los niños no toleran procedimientos endoscópicos con sedación mínima o moderada, por lo que la sedación debe ser profunda y en ocasiones requieren de anestesia general. Los pacientes menores de seis meses de edad presentan la mayor tasa de complicaciones respiratorias, por lo tanto se requiere anestesia general con IOT para el procedimiento ${ }^{81}$. Los adolescentes pueden tolerar los procedimientos endoscópicos con sedación leve, pero los niños, en especial menores de 10 años pueden no cooperar y no tolerar este procedimiento ${ }^{82}$.

Se recomienda que los procedimientos de niños con problemas médicos complejos, vía aérea difícil anticipada, obesidad mórbida y problemas de comportamiento se realicen en quirófano ${ }^{81,83}$.

Las sedaciones en el área de endoscopia pueden realizarse con la ayuda de combinaciones de ciertos medicamentos: ketamina-midazolam, fentanilo-propofol, midazolam-propofol. En los procedimientos realizados con ketamina y midazolam el paciente presenta mejor confort, pero la recuperación es más rápida y mejor con fentanilo y propofol $^{91,92}$.

En la colonoscopia podemos seguir los lineamientos mencionados anteriormente, ya que el acceso a la vía aérea no se ve obstaculizado, la sedación profunda se puede lograr más fácilmente, y si ocurren problemas respiratorios, las intervenciones en las vías respiratorias son fáciles de manejar ${ }^{81}$.

La evaluación de la vía aérea superior en los niños con endoscopio flexible o rígido presenta riesgo potencial de laringoespasmo y compromiso de las vías respiratorias, dicha evaluación generalmente se realiza en el quirófano bajo anestesia general ${ }^{93}$.

Las infecciones respiratorias en niños con vías respiratorias hiperreactivas son una contraindicación absoluta para la endoscopia electiva con sedación; en procedimientos de urgencia se requiere anestesia general ${ }^{85}$.

\section{Cuidados preprocedimiento, transprocedimiento y posprocedimiento en pacientes embarazadas}

\section{INDICACIONES DE PROCEDIMIENTOS ENDOSCÓPICOS DURANTE EL EMBARAZO}

La AMEG recomienda realizar los procedimientos endoscópicos durante el embrazo solo cuando la indicación sea clara y fuerte. Preferentemente todos los procedimientos habrá que diferirlos o realizarlos en el segundo trimestre del embarazo.

Los riesgos potenciales de los procedimientos anestésicos durante el embarazo incluyen la sobresedación que causa hipotensión e hipoxia en la madre, lo que puede provocar hipoxia en el feto e incluso conducir a la muerte fetal. Otros riesgos para el feto son la teratogénesis y el parto pretérmino. Es por ello que se sugiere diferir los procedimientos endoscópicos durante el embarazo.

Se estima que anualmente mas de 12,000 embarazadas en EE.UU. tienen patologías que requieren estudio de EGD. Otras 6,000 requieren colonoscopia y aproximadamente 1,000 tienen coledocolitiasis sintomática que requiere CPRE.

Alrededor del $79 \%$ de los procedimientos en el embarazo son diagnósticos y el resto terapéuticos ${ }^{4,9}$. Los riesgos, beneficios e indicaciones de los procedimientos endoscópicos durante el embarazo son poco conocidos. La mayoría de las recomendaciones actuales se basan en series de casos y reportes de caso ${ }^{20}$.

Los procedimientos invasivos durante el embarazo se justifican cuando al no realizarlos se pone en riesgo la vida del binomio madre-feto. Se ha reportado que la colonoscopia es segura durante el embarazo cuando hay una indicación fuerte ${ }^{86,87}$.

La sedación durante el primer trimestre está asociada a riesgo teratogénico y en el tercer trimestre a parto 
Tabla 9. Indicaciones de endoscopia en el embarazo Hemorragia gastrointestinal con inestabilidad hemodinámica

Náuseas, vómitos o dolor abdominal severo o refractario a tratamiento

Odinofagia o disfagia

Sospecha fuerte de neoplasia maligna de colon

Diarrea severa con pruebas diagnósticas negativas

Pancreatitis biliar con coledocolitiasis sintomática o colangitis

Lesión del conducto biliar o pancreático

prematuro. La exposición a cualquier procedimiento endoscópico se asocia con mayor riesgo de parto prematuro(RR: 1.54; IC 95\%: 1.36-1.75) y a talla baja para la edad gestacional (RR: 1.45; IC 95\%: 0.87-2.4) ${ }^{88}$. Por ello se recomienda diferir hasta el término del embarazo o bien si es posible y está indicado, realizarlo durante el segundo trimestre del embarazo ${ }^{89}$ (Tabla 9).

\section{Dosis y medicamentos categorías A y B EN PACIENTES EMBARAZADAS}

La AMEG recomienda seguir los lineamientos emitidos por la Administración de Alimentos y Fármacos (FDA) de los EE.UU. y del Colegio Americano de Obstétras y Ginecólogos en relación con la utilización de fármacos categoría $B$ durante el embarazo, procurando un tiempo de exposición mínimo e inducción minimizada.

Cuando no se puedan diferir, los procedimientos anestésicos durante el embarazo requieren un anestesiólogo especialmente capacitado y endoscopistas experimentados que permitan disminuir al máximo la exposición y riesgos potenciales de los fármacos y el procedimiento sobre el binomio madre-feto ${ }^{90-92}$.

De acuerdo con la FDA ${ }^{93}$, los sedantes que se consideran categoría $B$, es decir, seguros o de bajo riesgo durante el embarazo incluyen el propofol y los opiáceos (Tabla 10).

Las ventajas del propofol sobre otros fármacos es su tiempo más corto de inicio, mayor profundidad de la sedación y menor tiempo de recuperación ${ }^{91-94}$.

En un estudio en ovejas embarazadas a las que se les administró propofol durante el tercer trimestre del embarazo, no se observaron diferencias significativas en la función cardiopulmonar materna y fetal. La función respiratoria del feto puede verse comprometida cuando se utilizan dosis máximas y el tiempo anestésico es prolongado ${ }^{95-97}$.

El uso de fentanilo y midazolam intravenosos antes del parto a dosis bajas (fentanilo $1 \mu \mathrm{g} / \mathrm{kg}$ y midazolam $0.02 \mathrm{mg} / \mathrm{kg}$ ) no modifican las puntuaciones en las escalas neonatales (APGAR, $\mathrm{SatO}_{2}$ ), lo que sugiere que dosis mínimas de fentanilo son seguras durante procedimientos en el embarazo ${ }^{96-99}$.

\section{MONITORIZACIÓN Y ADMINISTRACIÓN DE OXÍGENO EN PACIENTES EMBARAZADAS}

La AMEG recomienda monitorización materno-fetal y administración de oxígeno antes, durante y posterior a los procedimientos endoscópicos realizados durante el embarazo.

La información sobre el monitoreo fetal durante un procedimiento endoscópico se extrapola de las recomendaciones del Colégico Americano de Obstétras y Ginecólogos (CAOG) y de la ASA sobre procedimientos quirúrgicos no obstétricos durante el embarazo ${ }^{100,101 .}$

El CAOG y la ASA recomiendan que si el feto se considera previable (antes de las 28 semanas de gestación) es suficiente comprobar la FC fetal utilizando Doppler antes y después del procedimiento. Si se considera que el feto será viable, se debe realizar monitorización simultánea de la FC fetal y de las contracciones uterinas antes y después del procedimiento ${ }^{100,101}$.

Con relación a la suplementación de oxígeno durante los procedimientos endoscópicos deben seguirse las recomendaciones previamente descritas en este documento.

\section{Posición materna duRANTE el PROCEDIMIENTO ENDOSCÓPICO REALIZADO DURANTE EL EMBARAZO}

La AMEG sugiere colocar al paciente en decúbito lateral izquierdo durante los procedimientos endoscópicos realizado durante el embarazo.

Durante el segundo y especialmente en el tercer trimestre del embarazo, el útero puede aplicar presión sobre la aorta y la vena cava y causar el síndrome de compresión aorta-cava. Este último puede disminuir la precarga, el flujo sanguíneo uterino y la perfusión placentaria, provocando hipoxia fetal ${ }^{102}$.

La posición en decúbito lateral izquierdo previene hipoxia e hipotensión durante un procedimiento 
Tabla 10. Fármacos utilizados para sedación y analgesia en endoscopia durante el embarazo y su categoría de riesgo según la Food and Drug Administration (FDA)

\begin{tabular}{|c|c|c|c|}
\hline $\begin{array}{l}\text { Categoría } \\
\text { FDA en el embarazo }\end{array}$ & Fármaco & $\begin{array}{l}\text { Resumen de la seguridad del fármaco reportada en } \\
\text { la literatura }\end{array}$ & Efectos secundarios \\
\hline A & & $\begin{array}{l}\text { Adecuado, buenos estudios controlados en mujeres } \\
\text { embarazadas que no muestran riesgo incrementado } \\
\text { de anormalidades fetales }\end{array}$ & \\
\hline \multirow[t]{4}{*}{ B } & Meperidina & $\begin{array}{l}\text { Datos en humanos sugieren algún riesgo si se usa } \\
\text { de forma prolongada o en dosis máximas }\end{array}$ & $\begin{array}{l}\text { Hipotensión, interacción con } \\
\text { inhibidores de la MAO }\end{array}$ \\
\hline & Propofol & $\begin{array}{l}\text { Datos limitados en humanos. Datos en animales } \\
\text { sugieren bajo riesgo }\end{array}$ & $\begin{array}{l}\text { Depresión respiratoria, hipotensión, } \\
\text { dolor en sitio de inyección }\end{array}$ \\
\hline & Ketamina & $\begin{array}{l}\text { Datos limitados en humanos. Datos en animales } \\
\text { sugieren bajo riesgo }\end{array}$ & $\begin{array}{l}\text { Depresión cardiorrespiratoria mínima, } \\
\text { reflejos protectores preservados }\end{array}$ \\
\hline & Naloxona & Probablemente seguro en estudios en humanos & \\
\hline \multirow[t]{2}{*}{ C } & Fentanilo & $\begin{array}{l}\text { Datos en humanos sugieren algún riesgo cuando es } \\
\text { usado en el tercer trimestre }\end{array}$ & $\begin{array}{l}\text { Depresión respiratoria, tórax leñoso } \\
\text { cuando se administra de forma rápida }\end{array}$ \\
\hline & Flumazenilo & $\begin{array}{l}\text { Datos limitados, estudios en animales sugieren bajo } \\
\text { riego }\end{array}$ & \\
\hline \multirow[t]{2}{*}{$D$} & Diazepam & $\begin{array}{l}\text { Datos en humanos sugieren posibles riesgos si se } \\
\text { usa en el primer y tercer trimestres del embarazo }\end{array}$ & Depresión cardiorrespiratoria \\
\hline & Midazolam & $\begin{array}{l}\text { Estudios limitados en humanos. Datos en animales } \\
\text { sugieren bajo riesgo }\end{array}$ & Depresión cardiorrespiratoria \\
\hline
\end{tabular}

MA0: monoaminooxidasa.

Adaptada de Savas,2014 ; Neuman, et al., 201396, FDA/CDER SBIA, 201997; Capell, 2011101; Shah, et al., $2019^{102}$

endoscópico en pacientes embarazadas en el tercer trimestre, por lo que es la posición en la que debe colocarse a la madre.

\section{Cuidados preprocedimiento, transprocedimiento y posprocedimiento en el paciente con obesidad grave y el paciente geriátrico}

La AMEG recomienda que se tomen consideraciones específicas para determinar el tipo de procedimiento anestésico, medicamentos, así como los aditamentos que utilizar en pacientes ancianos y obesos en quienes se realizará un procedimiento endoscópico.

Los cuidados preoperatorios, transoperatorios y postoperatorios para los procedimientos endoscópicos en el paciente geriátricos y obesos no difieren a los de otros grupos de edad. Las complicaciones relacionadas con los procedimientos anestésicos más frecuentes en este grupo de pacientes son bradicardia, hipotensión y disminución de la $\mathrm{SatO}_{2}$.

El uso de propofol en mayores de 75 años no se asocia a mayores efectos adversos como: hipoxemia
$\left(\mathrm{SatO}_{2}<90 \%\right)$, bradicardia ( $\mathrm{FC}<40-50 \mathrm{lpm}$ ) e hipotensión (presión arterial sistólica $<90 \mathrm{mmHg}$ ), comparado con pacientes $<75$ años. La frecuencia estimada de estos eventos es menor al $5 \%{ }^{102}$.

La frecuencia de eventos como hipotensión, desaturación, bradicardia, hipertensión, arritmia y aspiración en pacientes sometidos a sedación continua con propofol es del $0.1 \%{ }^{103}$.

Aunque los pacientes obesos tienen mayor riesgo de complicaciones como hipoxemia, no se han observado diferencias en la frecuencia de esta o de otras complicaciones respiratorias cuando se administra oxígeno mediante puntas nasales o con mascarilla nasal con presión positiva (MNPP).

En pacientes con obesidad grado II se recomienda el uso de mascarilla nasal con presión positiva durante el procedimiento o incluso como medida para solucionar o mejorar el estado de hipoxemia (saturación menor al $90 \%$ ) que pueda ocurrir antes, durante o posterior al procedimiento. El uso de MNPP es comparable con métodos de ventilación invasiva (p. ej., IOT o mascarilla laríngea $)^{103}$, aunque la IOT en pacientes de alto riesgo disminuye significativamente la aparición de complicaciones relacionadas con la sedación ${ }^{104}$. 


\section{Financiamiento}

La presente investigación no ha recibido ninguna beca especifica de agencias de los sectores público, comercial, o sin ánimo de lucro.

\section{Conclusiones}

El presente posicionamiento es el resultado de un análisis multidisciplinario basado en la evidencia disponible hasta el momento de su elaboración y en la opinión de especialistas expertos. Tiene la intención de proveer recomendaciones que ayuden a mejorar y mantener la calidad de la sedación en los procedimientos endoscópicos.

\section{Conflicto de intereses}

La Dra. Alejandra Noble Lugo es conferencista de Asofarma, Menarini y Takeda. El resto de los participantes negaron tener algún conflicto de interés o relación financiera que declarar.

\section{Responsabilidades éticas}

Protección de personas y animales. Los autores declaran que para esta investigación no se han realizado experimentos en seres humanos ni en animales.

Confidencialidad de los datos. Los autores declaran que en este artículo no aparecen datos de pacientes.

Derecho a la privacidad y consentimiento informado. Los autores declaran que en este artículo no aparecen datos de pacientes.

\section{Bibliografía}

1. Tohda G, Higashi S, Wakahara S, Morikawa M, Sakumoto H, Kane T. Propofol sedation during endoscopic procedures: Safe and effective administration by registered nurses supervised by endoscopists. Endoscopy. 2006;38(4):360-7.

2. NORMA Oficial Mexicana NOM-006-SSA3-2011, Para la práctica de la anestesiología. Diario oficial de la federación, DOF: 23/03/2012.

3. ASGE Standards of Practice Committee. Early DS, Lightdale JR, Vargo JJ, Acosta RD, Chandrasekhara V, Chathadi KV, et al. Guidelines for sedation and anesthesia in GI endoscopy. Gastrointest Endosc. 2018;87:327-37.

4. Igea F, Casellas JA, González-Huix F, Gómez-Oliva C, Baudet J, Cacho G, et al. Sedación en endoscopia digestiva. Guía de práctica clínica de la Sociedad Española de Endoscopia Digestiva. Rev Esp Enferm Dig. 2014;3:195-211.

5. Practice Guidelines for Moderate Procedural Sedation and Analgesia 2018: A Report by the American Society of Anesthesiologists. Anesthesiology. 2018;128:437-79.

6. Lin OS. Sedation for routine gastrointestinal endoscopic procedures: a review on efficacy, safety, efficiency, cost and satisfaction. Intest Res. 2017; 15:456-66.

7. Cohen LB, Wecsler JS, Gaetano JN, Benson AA, Miller KM, Durkalski V, et al. Endoscopic sedation in the United States: results from a nationwide survey. Am J Gastroenterol. 2006;101:967-74.
8. Ghanouni A, Plumb A, Hewitson P, Nickerson C, Rees CJ, von Wagner C. Patients' experience of colonoscopy in the English Bowel Cancer Screening Programme. Endoscopy. 2016;48:232-40.

9. Radaelli F, Meucci G, Sgroi G, Minoli G; Italian Association of Hospital Gastroenterologists (AIGO). Technical performance of colonoscopy: the key role of sedation/analgesia and other quality indicators. Am J Gastroenterol. 2008;103(5):1122-30.

10. Aisenberg J, Cohen LB, Piorkowski JD. Propofol use under the direction of trained gastroenterologists: an analysis of the medicolegal implications. Am J Gastroenterol. 2007;101:707-13.

11. Cohen L, Delegge M, Aisenberg J, Brill JV, Inadomi JM, Kochman ML, et al.; AGA institute review of endoscopic sedation. Gastroenterology. 2007;133:675-701

12. Vargo JJ, Niklewski PJ, Williams JL, Martin JF, Faigel DO. Patient safety during sedation by anesthesia professionals during routine upper endoscopy and colonoscopy: an analysis of 1.38 million procedures. Gastrointest Endosc. 2017;85(1):101-8.

13. Jackson $D$, Johnson $B S$. Conscious sedation for dentistry: risk management and patient selection. Dent Clin N Am. 2002:46;767-80.

14. Diaz LK, Jones L. The child with congenital heart disease. Anesthesiology Clin. 2009;27(2):301-19.

15. Carey E, Sorbi D. Unsedated endoscopy. Gastrointest Endoscopy. Clin N Am. 2004;14:369-83.

16. Sami SS, Subramanian V, Ortiz-Fernández-Sordo J, Saeed A, Singh S, Guha IN, et al. Performance characteristics of unsedated ultrathin video endoscopy in the assessment of the upper GI tract: systematic review and meta-analysis. Gastrointest Endosc. 2015;82:782-92.

17. Leung FW. Water-aided colonoscopy. Gastroenterol Clin North Am. 2013:42:507-19.

18. Leung F, Harker J, Jackson G. A proof of principle, prospective, randomized, controlled trial demostrating improved outcomes in scheduled unsedated colonoscopy by the water method. Gastrointest Endoscopy. 2010;72(4):693-700

19. Rex DK, Imperiale TF, Portish V. Patients willing to try colonoscopy without sedation: associated clinical factors and results of a randomized controlled trial. Gastrointest Endosc. 1999;49:554-9.

20. Neena A, Fallone C, Mayrand S, Huang J, Wieczorek P, Barkun A. Sedation versus no sedation in the performance of diagnostic upper gastrointestinal endoscopy. A Canadian randomized controlled cost-outcome study. Am J Gastroenterol. 2004;99:1692-9.

21. McQuaid K, Laine L. A systematic review and meta-analysis of randomized, controlled trials of moderate sedation for routine endoscopic procedures. Gastrointest Endosc. 2008;67:910-92.

22. Sharp C, Tayler E, Ginsberg G. Anesthesia for routine and advanced upper gastrointestinal endoscopic procedures. Anesthesiology Clin. 2017;35:669-77.

23. Sidhu R, Turnbull D, Newton M, Thomas-Gibson S, Sanders DS, Hebbar S, et al. Deep sedation and anaesthesia in complex gastrointestinal endoscopy: a joint position statement endorsed by the British Society of Gastroenterology (BSG), Joint Advisory Group (JAG) and Royal College of Anaesthetists (RCOA). Frontline Gastroenterol. 2019;10:141-7.

24. Goudra B, Singh PM. Critical analysis of guidelines for providing sedation to patients undergoing gastrointestinal endoscopy procedures. Anesth Essays Res. 2019;13:601-7.

25. Shah A, Bohra S, Shah M. Sedation and anesthesia in gastrointestinal endoscopy: Indian scenario. J Digest Endosc. 2019;10:97-100.

26. Padmanabhan A, Frangopoulos Ch, Shaffer L. Patient satisfaction whit propofol for outpatient colonoscopy: A prospective, randomized, double blind study. Dis Colon Rectum. 2017;60(10):1102-8.

27. Keating GM. Dexmedetomidine: A review of its use for sedation in the intensive care setting. Drugs. 2015;75(10):1119-30.

28. Goudra B, Gouda G, Mohinder P. Recent developments in drugs for GI endoscopy sedation. Dig Dis Sci. 2020;65(10):2781-8.

29. Kopacova M, Bures J. Informed consent for digestive endoscopy. World $\mathrm{J}$ Gastroeintest Endosc. 2012;4(16).

30. Triantafyllou K, Stanciu C, Kruse A, Malfertheiner P, Axon A, Ladas SD European Society of Gastrointestinal Endoscopy. Informed consent for gastrointestinal endoscopy: a 2002 ESGE survey. Dig Dis. 2002;20(3-4):280-3.

31. De Silva AP, Amarasiri L, Liyanage MN, Kottachchi D, Dassanayake AS, De Silva HJ. One-hour fast for water and six-hour fast for solids prior to endoscopy provides good endoscopic vision and results in minimum patient discomfort. J Gastroenterol Hepatol. 2009;24(6):1095-7.

32. Barkun AN, Bardou M, Martel M, Gralnek IM, Sung JJY. Prokinetics in acute upper Gl bleeding: A meta-analysis. Gastrointest Endosc. 2010; 72(6):1138-45

33. Rehman A, Iscimen R, Yilmaz M, Khan H, Belsher J, Gomez JF, et al. Prophylactic endotracheal intubation in critically ill patients undergoing endoscopy for upper GI hemorrhage. Gastrointest Endosc. 2009;69(7):e55-9.

34. Kane-Gill SL, Olsen KM, Rebuck JA, Rea RS, Boatwright DW, Smythe MA, et al. Multicenter treatment and outcome evaluation of aspiration syndromes in critically ill patients. Ann Pharmacother. 2007; 41(4):549-55. 
35. Yoo YC. Endoscopic sedation: Risk assessment and monitoring. Clin Endosc. 2014;47(2):151-4

36. Triantafillidis JK, Merikas E, Nikolakis D, Papalois AE. Sedation in gastrointestinal endoscopy: Current issues. World J Gastroenterol. 2013;19(4):463-81.

37. Chang B, Urman R. Non-operating room anesthesia, the principles of patient assesment and preparation. Anesthesiology Clin. 2016;34:223-40.

38. Committee on Standards and Practice Parameters; Apfelbaum JL, Connis RT, Nickinovich DG, American Society of Anesthesiologists Task Force on Preanesthesia Evaluation; Pasternak LR, Arens JF, et al. Practice Advisory for preanesthesia evaluation. An Update report by the American Society of Anethesiologist Task Force on Preanethesia evaluation. Anesthesiology. 2012;116(3):522-38.

39. Geissler HJ. Risk stratification in heart surgery: comparison of six score systems. Eur J Cardiothorac Surg. 2000:17:400-6.

40. Baretti R, Pannek N, Knecht JP, Krabatsch T, Hübler S, Htzer R. Risk stratification of morbidity and mortality in coronary artery bypass surgery. Thorac Cardiov Surg. 2002;50:237-46.

41. Peña B, Enrique J. La evaluación preoperatoria con riesgo anestésico. Rev Colomb Anestesiol. 2007;35(2):97-9

42. Haynes AB, Weiser TG, Berry WR, Lipsitz SR, Breizat AH, Dellinger EP et al. A surgical safety checklist to reduce morbidity and mortality in global population. N Engl J Med. 2009;360:491-9.

43. Mason MC, Griggs RK, Withecombe R, Xing EY, Sandberg C, Molyneux MK Improvement in staff compliance with a safety standard checklist in endoscopy in a tertiary center. BMJ Open Qual. 2018; 7(3):e000294.

44. Matharoo M, Thomas-Gibson S, Haycock A. Implementation of an endoscopy safety checklist. Frontline Gastroenterology. 2014;5:260-5.

45. Amornyotin S. Sedation-related complications in gastrointestinal endoscopy. World J Gastrointest Endosc. 2013;5:527-33.

46. Bell GD, Morden A, Bown S, Coady T, Logan RF. Prevention of hipoxaemia during upper-gastrointestinal endoscopy by means of oxygen via nasal cannulae. Lancet. 1987;1:1022-24.

47. ASGE Standards of Practice Committee. Early DS, Lightdale JR, Vargo JJ Acosta RD, Chandrasekhara V, Chathadi KV, et al. Guidelines for sedation and anesthesia in Gl endoscopy. Gastrointest Endosc. 2018;87:327-37.

48. Demonceau JM, Riphaus A, Aparicio JR, Beilenhoff U, Knape JT, Ortmann M, et al. European Society of Gastrointestinal Endoscopy, European Society of Gastroenterology and Endoscopy Nurses and Associates, and the European Society of Anesthesiology Guideline: Non-anesthesiologist administration of Propofol for GI endoscopy. Endoscopy. 2010:42:960-74.

49. Conway A, Douglas C, Sutherland JR. A systematic review of capnography for sedation. Anesthesia. 2016;71:450-4.

50. Kim SH, Park M, Lee J, Kim E, Choi YS. The addition of capnography to standard monitoring reduces hypoxemic events during gastrointestinal endoscopic sedation: a systematic review and meta-analysis. Ther Clin Risk Manag. 2018;14:1605-14.

51. Riphaus A, Wehrmann T, Kronshage T, Geist C, Pox CP, Heringlake S, et al. Clinical value of the Integrated Pulmonary Index® during sedation for interventional upper GI-endoscopy: A randomized, prospective tri-center study. Dig Liver Dis. 2017;49:45-9.

52. Hernández-Gancedo $C$, Pestaña D, Pérez-Chrzanowska H, Martínez-Casanova E, Criado A. Comparing entropy and the Bispectral Index with the Ramsay Score in sedated ICU patients. J Clin Monit Comput. 2007:21:295-302

53. Von Delius S, Salletmaier H, Meining A, Wagenpfeil S, Saur D, Bajbouj $\mathrm{M}$, et al. Bispectral index for monitoring sedation during endoscopic retrograde cholangiopancreatography: a randomized clinical trial (the EndoBIS study). Endoscopy. 2012;44:258-64.

54. Park S, Lee H, Ahn H. Bispectral Index versus standard monitoring in sedation for endoscopic procedures: A systematic review and meta-analysis. Dig Dis Sci. 2016:61(3):814-24.

55. Kotwinski D, Paton L, Langford R. The role of high flow nasal oxygen therapy in anaesthesia. Br J Hosp Med (Lond). 2018;79:620-7.

56. Nishimura M. High-flow cannula oxygen therapy in adults: Physiological benefits, indication, clinical benefits and adverse effects. Respir Care. 2016;61:529-451

57. Masclans JR, Pérez-Teran P, Roca O. Papel de la oxigenoterapia de alto flujo en la insuficiencia respiratoria aguda. Med Intensiva. 2015;39:505-15.

58. Douglas N, Nazeem F, Lee K, Mezzavia P, Krieser R, Steinfort D, et al. $A$ randomized controlled trial comparing high-flow nasal oxygen sith standard management for conscious sedation during bronchoscopy. Anaesthesia. 2018;73:169-76.

59. Schumann R, Natov N, Rocuts-Martinez K, Finkelman MD, Phan TV, Hegde SR, et al. High-flow nasal oxygen availability for sedation decreases the use of general anesthesia during endoscopic retrograde cholangiopancreatography and endoscopic ultrasound. World J Gastroenterol. 2016;22:10398-405.

60. Yuxuan L, Xiaoging Z, Lizhi L, Wei M, Zhao B, Wang X, et al. High-flow nasal cannula oxygen therapy and hypoxia during gastroscopy with propofol sedation: a randomized multicenter clinical trial. Gastrointest Endosc. 2019;90:591-601.
61. Benatar Puente F, Galaviz Oñate DA, Chávez Ramírez MA, Olivares Mendoza $\mathrm{H}$. Terapia nasal de alto flujo versus administración convencional de oxígeno en pacientes sometidos a endoscopia gastrointestinal. An Med (Mex). 2017;62:187-91.

62. Riccio C, Sarmiento S, Minhujuddin A. High-flow versus standard nasal cannula in morbidly obese patients during colonoscopy: A prospective, randomized clinical trial. J Clin Anesth. 2019:54:19-24.

63. ASGE. Quality indicators common to all GI endoscopic procedures. Gastrointest Endosc. 2015;81(1):3-16.

64. ASGE Ensuring Safety in the Gastrointestinal Endoscopy Unit Task Force, Calderwood AH, Chapman FJ, Cohen J, Cohen LB, Collins J, Day LW, et al. Guidelines for safety in the gastrointestinal endoscopy unit. Gastrointest Endosc. 2014;79(3):363-72.

65. Satyen P, Pankaj K, Mohan VK, Sandeep K. Standars of care for procedural sedation: Focus on differing perceptions among societies. Indian J Anaesth. 2018;62(7):493-6.

66. Practice Guidelines for Moderate Procedural Sedation and Analgesia 2018: A Report by the American Society of Anesthesiologists Task Force on Moderate Procedural Sedation and Analgesia, the American Association of Oral and Maxillofacial Surgeons, American College of Radiology, American Dental Association, American Society of Dentist Anesthesiologists and Society of Intervention Radiology. Anesthesiology. 2018;128(3):1-43.

67. Practice Guidelines for Postanesthetic Care. An Update Report by the American Society of Anesthesiologists Task Force on Postanesthetic Care. Anesthesiology. 2013;18: 291-317.

68. American Society of Anesthesiologists Committee. Practice Guidelines for Preoperative Fasting and the Use of Pharmacologic Agents to Reduce the Risk of Pulmonary Aspiration: Application to Healthy Patients Undergoing Elective Procedures. Anesthesiology. 2011;114(3):495-511.

69. Andersson H, Schmitz A, Frykholm P. Preoperative fasting guidelines in pediatric anesthesia: are we ready for a change? Pediatric Anesthesia. 2018;31(3):342-8.

70. Andersson $\mathrm{H}$, Hellstrom $\mathrm{P}$, Frykholm $\mathrm{P}$. Introducing the 6-4-0 fasting regimen and the incidence of prolonged preoperative fasting in children. Pediatric Anesthesia. 2018;28:46-52.

71. Fawcett W, Thomas M. Pre-operative fasting in adults and children: clinical practice and guidelines. Anaesthesia. 2019;74:83-8.

72. Thomas M, Morrison C, Newton R, Schindler E. Consensus statement on clear fluids fasting for elective pediatric general anesthesia. Pediatric Anesthesia. 2018;28:411-4.

73. Moura LA, Dias IMG, Pereira LV. Prevalence and factors associated with preoperative anxiety in children aged 5-12 years. Rev Latino-Am Enfermagem. 2016;24:e2708.

74. Domínguez CER. Dexmedetomidina vía oral y vía nasal en pacientes de 1 a 5 años. Evid Med Invest Salud. 2014;7(S1):S14-S16.

75. Riera C, González CG, Vaca C, Marchant P, Glenz C, Larraín F, et al. Sedación, seguridad y utilidad de la endoscopia digestiva alta en pediatría. Rev Chil Pediatr. 2010;81(1):37-45.

76. Khalila A, Shavit I, Shaoul R. Propofol sedation by pediatric gastroenterologists for endoscopic procedures: A retrospective analysis. Front $\mathrm{Pe}-$ diatr. 2019;7(98):1-7.

77. Linares Segovia B, García Cuevas MA, Ramírez Casillas IL, Guerrero Romero JF, Botello Buenrostro I, Monroy Torres R, et al. Medicación preanestésica con dexmedetomidina intranasal y midazolam oral como ansiolítico. Un ensayo clínico. An Pediatr (Barc). 2014;81(4):226-31.

78. Restrepo A, Villalobos MA, González de Cosío C, Gutiérrez L, Sánchez R, Espinosa G, et al. Prevención de la hipoxia al administrar oxígeno por boquilla durante el estudio endoscópico con sedación. Endoscopia (Mex). 1999;10(2):45-8.

79. Smith Z, Mullady DK, Lang G, Koushik K, Hovis RM, Patel RS, et al. A randomized controlled trial evaluating general endotracheal anesthesia versus monitored anesthesia care and the incidence of sedation-related adverse events during ERCP in high-risk patients. Gastrointest Endosc. 2019;89(4):855-62

80. Rodríguez NE. Manejo anestésico de procedimientos endoscópicos en pediatría. Rev Mex Anestesiol. 2010;33:79-85.

81. Mason K, Holzman R. Anesthesia and sedation for pediatric procedures outside the operating room. En: Davis PJ, Cladis FP, Motoyama EK. Smith's Anesthesia for Infants and Children. Philadelphia: Elsevier; 2011. pp. 1041-1057.

82. Akbulut U, Saylan S, Sengu B, Akcali GE, Erturk E, Cakir M. A comparison of sedation with midazolam-ketamine versus propofol-fentanyl during endoscopy in children: a randomized trial. Eur J Gastroenterol Hepatol. 2017;29(1):112-8.

83. Edwards C, Kapoor C, Samuel C, Issenman R, Brill H. General anesthetic versus light sedation: Effect on pediatric endoscopy wait times. Can J Gastroenterol. 2013;27(9):519-22.

84. Jatana KR, Rastatter JC. Endoscopy in the evaluation and management of the pediatric airway. En: Iancu C, editor. Advances in Endoscopic Surgery. InTech Open; 2011. Available from: https://www.intechopen. com/books/advances-in-endoscopic-surgery/endoscopy-in-the-evaluation-and-management-of-the-pediatric-airway 
85. Friedt M, Welsch S. An update on pediatric endoscopy. Eur J Med Res. 2013;18(24):1-7.

86. De Lima A, Galjart B, Wisse PHA, Bramer WM, van der Woude CJ. Does lower gastrointestinal endoscopy during pregnancy pose a risk for mother and child? -a systematic review. BMC Gastroenterol. 2015;15:15.

87. ASGE Standard of Practice Committee; Shergill AK, Ben-Menachem T, Chandrasekhara V, Chathadi K, Decker GA, Evans JA, et al. Guidelines for endoscopy in pregnant and lactating women. Gastrointest Endosc. 2012;76:18-24.

88. De Lima A, Zelinkova Z, van der Woude CJ. A prospective study of the safety of lower gastrointestinal endoscopy during pregnancy in patients with inflammatory bowel disease. J Crohns Colitis. 2015;9:519-24.

89. Ludvigsson JF, Lebwohl B, Ekbom A, Kiran RP, Green PH, Höijer J, et al. Outcomes of pregnancies for women undergoing endoscopy while they were pregnant: A nationwide cohort study. Gastroenterol. 2017; 152:554-63.

90. Savas N. Gastrointestinal endoscopy in pregnancy. World J Gastroenterol. 2014;20(41):15241-52.

91. Cappell MS. Sedation and analgesia for gastrointestinal endoscopy during pregnancy. Gastrointest. Endoscopy Clin N Am. 2006;16:1-31.

92. Neuman G, Koren G. Safety of procedural sedation in pregnancy. J Obstet Gynaecol Can. 2013;35(2):168-73.

93. FDA/CDER SBIA Chronicles. Drugs in Pregnancy and Lactation: Improved Benefit-Risk Information [Internet]. CDER Small Business and Industry Assistance (SBIA); 22/01/2015 [fecha de acceso: 01/08/2019]. Disponible en: https://www.fda.gov/files/drugs/ published/\%22Drugs-in-Pregnancy-and-Lactation--Improved-Benefit-Risk-Information\%22-January-22--2015-Issue.pdf

94. Nurten SA. Endoscopy in pregnant patients. Endoscopy. En: Amornyotin S, editor. Croacia: InTech; 2013. pp. 321-348.
95. Andaluz A, Trasserras O, García F. Maternal and fetal effects of propofol anaesthesia in the pregnant ewe. Veterin J. 2005;170(1):77-83.

96. Frölich MA, Burchfield DJ, Euliano TY, Caton D. A single dose of fentanyl and midazolam prior to Cesarean section have no adverse neonatal effects. Can J Anesth. 2006;53(1):79-8.

97. Cappell M. Risks versus benefits of gastrointestinal endoscopy during pregnancy. Nat Rev Gastroenterol Hepatol. 2011;8(11):610-34.

98. Singh S. Review of endoscopy in pregnancy: Challenges and current evidence. J Gastrointest Dig Syst. 2018;8:3.

99. ACOG Committee Opinion No. 775. Nonobstetric surgery during pregnancy. Obstet Gynecol. 2019;133:e285-286.

100. Heesen M, Klimek M. Nonobstetric anesthesia during pregnancy. Curr Opin Anestthesiol. 2016;29:297-303.

101. Masaya N, Takuji G, Chika K, Masakatsu F, Takao I, Fuminori M. Safety of gastroenterologist-guided sedation with propofol for upper gastrointestinal therapeutic endoscopy in elderly patients compared with younger patients. Gut Liver. 2015;9(1):38-42.

102. Agostoni M, Fanti L, Gemma M, Pasculli N, Beretta L, Testoni PA. Adverse events during monitored anesthesia care for GI endoscopy: an 8-year experience. Gastrointest Endosc. 2011;74:266-75.

103. Gedeon M, Gomes S, Roy K, Duclos-Miller P, Rose JS. Use of noninvasive positive pressure ventilation in patients with severe obesity undergoing esophagogastroduodenoscopy: a randomized controlled trial. Surg Obes Relat Dis. 2019;15:1589-24.

104. Zachary IS, Daniel KM, Gabriel DL, Koushik K, Robert MH, Riddhi SP, et al. A randomized controlled trial evaluating general endotracheal anesthesia vesus monitored anesthesia care and the incidence of sedation-related adverse event during ERCP in high risk patients. Gastrointest Endosc. 2019;89(4):855-62. 